\title{
Short period eclipsing binary candidates identified using SuperWASP ${ }^{\star}$
}

A. J. Norton ${ }^{1}$, S. G. Payne ${ }^{1}$, T. Evans ${ }^{1}$, R. G. West ${ }^{2}$, P. J. Wheatley ${ }^{3}$, D. R. Anderson ${ }^{4}$, S. C. C. Barros ${ }^{5}$, O. W. Butters ${ }^{2}$, A. Collier Cameron ${ }^{6}$, D. J. Christian ${ }^{5,9}$, B. Enoch ${ }^{6}$, F. Faedi ${ }^{5}$, C. A. Haswell ${ }^{1}$, C. Hellier ${ }^{4}$, S. Holmes ${ }^{1}$, K. D. Horne ${ }^{6}$, S. R. Kane ${ }^{10}$, T. A. Lister ${ }^{7}$, P. F. L. Maxted ${ }^{4}$, N. Parley ${ }^{6}$, D. Pollacco ${ }^{5}$, E. K. Simpson ${ }^{5}$, I. Skillen ${ }^{8}$, B. Smalley ${ }^{4}$, J. Southworth ${ }^{4}$, and R. A. Street ${ }^{7}$

1 Department of Physics and Astronomy, The Open University, Walton Hall, Milton Keynes MK7 6AA, UK e-mail: a.j.norton@open.ac.uk

2 Department of Physics and Astronomy, University of Leicester, Leicester LE1 7RH, UK

3 Department of Physics, University of Warwick, Coventry CV4 7AL, UK

4 Astrophysics Group, Keele University, Staffordshire ST5 5BG, UK

5 Astrophysics Research Centre, Main Physics Building, School of Mathematics \& Physics, Queen's University, University Road, Belfast BT7 1NN, UK

${ }^{6}$ School of Physics and Astronomy, University of St. Andrews, North Haugh, St. Andrews, Fife KY16 9SS, UK

7 Las Cumbres Observatory Global Telescope Network, 6740 Cortona Drive, Suite 102, Goleta, CA 93117, USA

8 Isaac Newton Group of Telescopes, Apartado de Correos 321, 38700 Santa Cruz de la Palma, Tenerife, Spain

9 Department of Physics and Astronomy, California State University, Northridge, CA 91330, USA

10 NASA Exoplanet Science Institute, Caltech, MS 100-22, 770 South Wilson Avenue, Pasadena, CA 91125, USA

Received 6 January 2011 / Accepted 28 January 2011

\section{ABSTRACT}

\begin{abstract}
We present light curves and periods of 53 candidates for short period eclipsing binary stars identified by SuperWASP. These include 48 newly identified objects with periods $<2 \times 10^{4} \mathrm{~s}(\sim 0.23 \mathrm{~d})$, as well as the shortest period binary known with main sequence components (GSC2314-0530 = 1SWASP J022050.85 + 332047.6) and four other previously known W UMa stars (although the previously reported periods for two of these four are shown to be incorrect). The period distribution of main sequence contact binaries shows a sharp cut-off at a lower limit of around $0.22 \mathrm{~d}$, but until now, very few systems were known close to this limit. These new candidates will therefore be important for understanding the evolution of low mass stars and to allow investigation of the cause of the period cut-off.
\end{abstract}

Key words. binaries: eclipsing - stars: individual: GSC2314-0530 - stars: individual: NY Vir - stars: individual: V1067 Her stars: individual: V1104 Her

\section{Introduction}

The primary aim of the SuperWASP photometric survey (Pollacco et al. 2006) is to search for transiting extrasolar planets. However, its wide field of view (two sets of eight cameras, each of which covers $7.8^{\circ} \times 7.8^{\circ}$ ) and its long base line (operating since 2004) mean that it is also well suited to discovering and characterising many types of stellar variability, across (almost) the whole sky (Norton et al. 2007; Payne et al. 2011). A SuperWASP count rate of 1 count $\mathrm{s}^{-1}$ is approximately equal to a $V$ magnitude of 15 and this marks the faint limit of the survey. At the current time (December 2010), the SuperWASP archive $^{1}$ (Butters et al. 2010) contains around 275 billion data points on about 30 million unique objects, obtained from over 8 million images. The typical observing cadence also means that SuperWASP is sensitive to variability periods from around tens of minutes to months.

SuperWASP is thus able to identify many hundreds of thousands of eclipsing binaries, pulsating stars, and stars displaying

\footnotetext{
* Figure 2 is only available in electronic form at http: //www . aanda.org

${ }^{1}$ http://www.wasp.le.ac.uk/public/
}

rotational modulations of all kinds. The particular focus of this paper is short period $(P<0.23 \mathrm{~d})$ eclipsing binary stars. Main sequence stars in such close orbits will appear as W Ursa Majoris systems.

Low mass dwarf stars are very common, but the way in which they evolve in close binary systems is poorly understood. It has been clear for a number of years that there is a short period cut-off for such systems around an orbital period of $\sim 0.22 \mathrm{~d}$ (Rucinski 2007). The reason for this period cut-off was originally thought to be due to stars reaching their fully convective limit (Rucinski 1992), but is now believed to be related to magnetic wind-driven angular momentum loss mechanisms, and hence linked to the finite age of the binary population (Stepien 2006), although details of the process are unclear.

The shortest period binary known with main sequence components is GSC2314-0530 which we identified using SuperWASP as having a period of $0.1926 \mathrm{~d}$ (Norton et al. 2007) and was shown to be coincident with a ROSAT X-ray source (1SWASP J022050.85 + 332047.6 = 1RXS J022050.7 + 332049). This object was subsequently modelled by Dimitrov \& Kjurkchieva (2010) using multi-colour photometry and radial velocity spectroscopy, who showed that it consisted of two stars 
of mass $0.51 M_{\odot}$ and $0.26 M_{\odot}$. Although the two stars are close, the best-fit model has the two stars under-filling their Roche lobes, and so the binary is detached.

The aim of the current work is to use the wide-field capabilities of the SuperWASP survey to search for short period W UMa star candidates, in order to increase the sample of such objects substantially and allow detailed investigation of their properties.

\section{Creation of the sample}

A purpose-written period searching code has been run on the entire SuperWASP archive. As explained in Norton et al. (2007), the period search comprised two techniques: a CLEANed power spectrum (Lehto 1997) and a folding technique using $\chi^{2}$ tests of phase-dispersion minimisation. Each light curve with more than 1000 data points was analysed for each object on a per camera basis (to minimise the effect of systematic differences between different cameras), and multiple significant periods (in some cases) were identified for each. The SuperWASP input catalogue is based on the USNO-B1.0 sample of over 1 billion stars, which extends down to magnitude $V \sim 21$. However, because the SuperWASP pixels are large (around 14") it is sometimes the case that multiple SuperWASP object apertures (each based on the position of a single USNO-B1.0 star) sample the variability from a single object. This can result in multiple, closely spaced SuperWASP objects displaying the same periodic signal. Also, despite using the SysRem algorithm (Tamuz et al. 2005) to reduce systematic contaminants in the light curves, it is apparent that many spurious periodic signals remain, particularly at harmonics of 1 sidereal day (i.e. at periods of 1 sidereal day $/ n$ where $n=2,3,4 \ldots 20$, etc.). Period ranges within about a minute either side of these harmonics were therefore flagged as likely spurious in the analysis.

This exercise yielded around 5600 periodic signals in the period range from about $125 \mathrm{~min}$ to $167 \mathrm{~min}(0.087 \mathrm{~d}$ to $0.116 \mathrm{~d})$, excluding those period ranges close to $1 / 9 \mathrm{~d}$ ( $\sim 160 \mathrm{~min}), 1 / 10 \mathrm{~d}$ ( 144 min) and 1/11 d ( 131 min). A further 17300 periodic signals were identified close to $1 / 9 \mathrm{~d} ; 14500$ signals close to $1 / 10 \mathrm{~d}$; and 9300 signals close to $1 / 11 \mathrm{~d}$. The distribution of the number of objects as a function of period within this range is shown in Fig. 1. Note that a single variable object may give rise to multiple signals if it is observed by multiple cameras, or if the variability is sampled by multiple apertures corresponding to closely separated stars in the input catalogue. Hence there are fewer unique periodic objects in this period range than indicated by the numbers quoted above.

Using the period search method described, contact binaries will generally give an identified period that corresponds to half the orbital period of the system. Hence, the period range chosen above covers a range from about $250 \mathrm{~min}(0.175 \mathrm{~d})$ to $333 \mathrm{~min}$ $(0.23 \mathrm{~d})$ for eclipsing binary orbital periods. This is therefore the period range of interest, spanning from just longer than the suggested period cut-off $(0.22 \mathrm{~d})$ to below the period of the shortest known system $(0.19 \mathrm{~d})$.

\section{Results}

Each of the 5600 light curves with periods outside the three "contaminated" period regions were examined by eye to pick out candidate eclipsing binaries. Most of these light curves displayed broadly sinusoidal modulation so are likely to represent some form of rotational variability. Many others showed asymmetric light curves (with narrow maxima and broad minima)
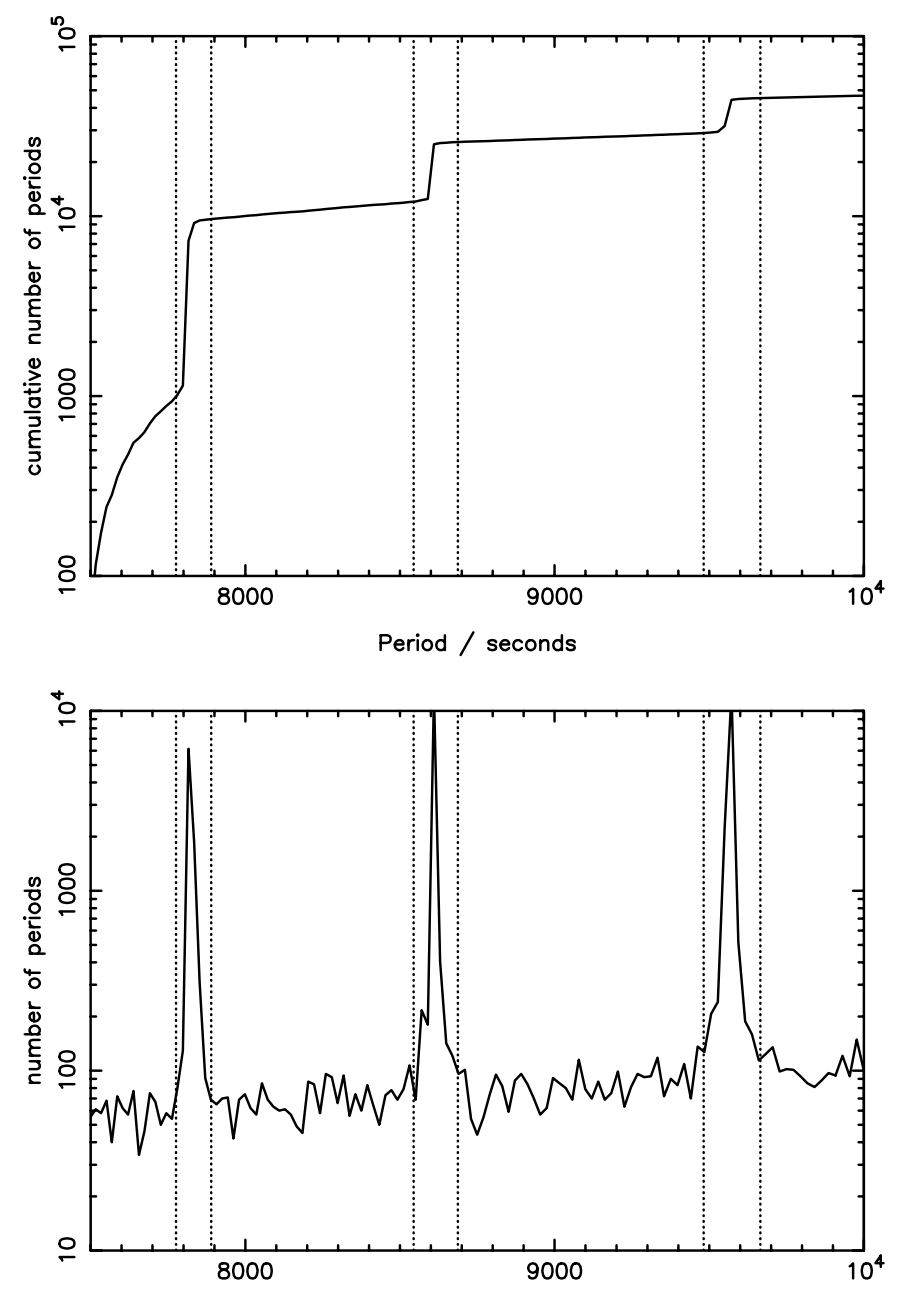

Fig. 1. The distribution of the periodically variable objects found in the period range from $125 \mathrm{~min}(7500 \mathrm{~s})$ to $167 \mathrm{~min}(10000 \mathrm{~s})$. Note the peaks at periods close to $1 / 11 \mathrm{~d}, 1 / 10 \mathrm{~d}$ and $1 / 9 \mathrm{~d}$ which are due to spurious signals caused by systematic noise. Periods within the ranges indicated by the dotted lines were flagged as likely spurious. Note also that contact binaries will typically give signals at half their true orbital period, so the period range here corresponds to contact binary orbital periods from $250 \mathrm{~min}$ to $333 \mathrm{~min}$.

characteristic of pulsating stars. These objects will be reported elsewhere. 40 unique objects, reported here, displayed light curves that are characteristic of W UMa stars, namely broad maxima with narrow minima (see Figs. 2, 3 and Table 1).

The large number of apparently periodic light curves close to periods of $1 / 9 \mathrm{~d}, 1 / 10 \mathrm{~d}$ and $1 / 11 \mathrm{~d}$ were not all eye-balled, as the vast majority of these are expected to be spurious signals due to systematic noise. However, the period distribution in Fig. 1 shows that $\sim 1 \%$ of these are likely to be genuine, so the $\sim 1 \%$ of them showing the strongest signals were examined individually. The strongest signals were chosen because the signal we are looking for is generally of higher amplitude than our typical systematic noise signal. This allowed a further 13 candidate eclipsing systems to be identified in the set of objects with periods close to $1 / 9 \mathrm{~d}$ (see Fig. 2 and Table 1). This exercise also revealed the known sdB+dM eclipsing binary, NY Vir (see Fig. 4), with a period of $145.463 \mathrm{~min}$, within the set of objects with periods close to $1 / 10 \mathrm{~d}$. No candidate W UMa stars were found in this latter set or in the set of objects close to periods of $1 / 11 \mathrm{~d}$, but the clear detection of NY Vir shows that such binaries would have been found, had they existed, despite the systematic noise 


\section{A. J. Norton et al.: WASP short period eclipsing binaries}
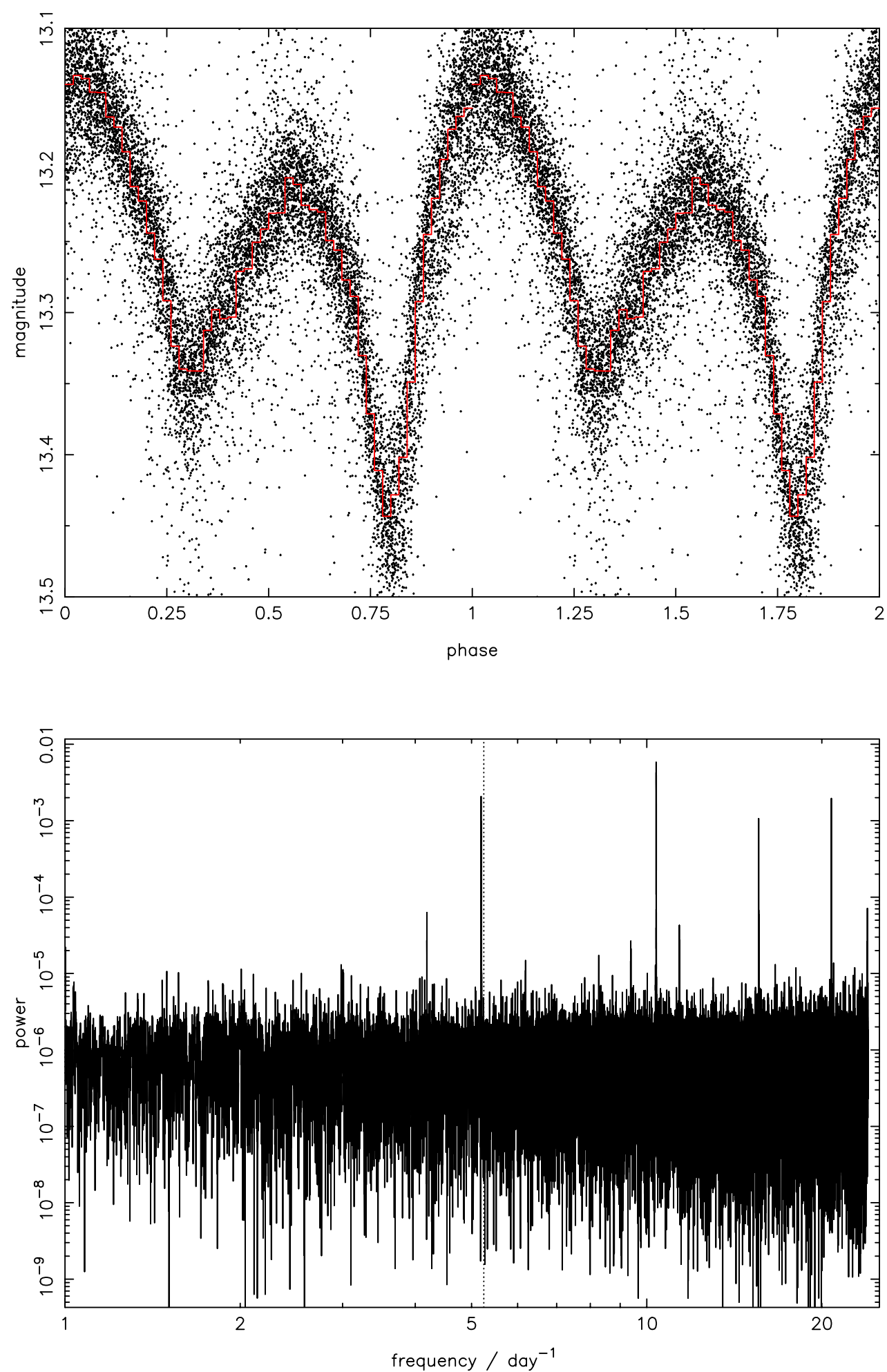

Fig. 3. (Top) The SuperWASP light curve of the shortest period binary known with dM components (GSC2314-0530 = 1SWASP J022050.85 + 332047.6 ), folded at a period of $277.395 \mathrm{~min}$. Phase zero corresponds to 2004 January 1st 00:00UT. The mean folded light curve (in 50 bins) is shown by an over-plotted line. (Bottom) The associated power spectrum, with the frequency corresponding to the orbital period indicated by a dotted line.

close to these periods. Figure 5 shows the orbital period distribution of the 53 candidate eclipsing binaries found and listed in Table 1.

Further evidence for the fact that these candidates are all $\mathrm{W}$ UMa stars is provided by their $V-K$ colours which are each significantly redder than the majority of the rest of the objects detected in this period range, as shown in Fig. 6. In fact, their $V-K$ colours of $\sim 1.5-3.5$ are typical of stars of spectral type K.

The 53 candidate eclipsing binaries include the known W UMa stars: V1067 Her, V1104 Her, ROTSE1 $\mathrm{J} 170240.11+151122.7$ and ASAS J111932-3950.8, as well as the shortest period object previously known, GSC2314-0530, 
Table 1. Candidate SuperWASP short period eclipsing binaries.

\begin{tabular}{|c|c|c|c|c|c|}
\hline $\begin{array}{l}\text { SuperWASP ID } \\
\text { 1SWASP Jhhmmss.ss } \pm \text { ddmmss.s }\end{array}$ & Period/day & $\begin{array}{l}\text { Maximum } \\
\text { SW V mag }\end{array}$ & $\begin{array}{l}\text { Primary } \\
\text { Depth }\end{array}$ & $\begin{array}{l}\text { Secondary } \\
\text { Depth }\end{array}$ & Other Name \\
\hline 1SWASP J052036.84+030402.1 & 0.23140 & 12.35 & 0.27 & 0.27 & \\
\hline 1SWASP J220734.47+265528.6 & 0.23123 & 14.25 & 0.24 & 0.22 & \\
\hline 1SWASP J000437.82+033301.2 & 0.23119 & 14.53 & 0.42 & 0.40 & \\
\hline 1SWASP J170240.07+151123.5 & 0.23119 & 13.79 & 0.42 & 0.39 & ROTSE1 J170240.11+151122.7 \\
\hline 1SWASP J041655.13-492709.8 & 0.23102 & 15.04 & 0.43 & 0.35 & \\
\hline 1SWASP J051501.18-021948.7 & 0.23090 & 13.78 & 0.15 & 0.15 & \\
\hline 1SWASP J235333.60+455245.8 & 0.23074 & 14.75 & 0.38 & 0.25 & \\
\hline 1SWASP J232607.07-294130.7 & 0.23012 & 13.71 & 0.39 & 0.37 & \\
\hline 1SWASP J155822.10-025604.8 & 0.23008 & 13.77 & 0.18 & 0.18 & \\
\hline 1SWASP J150822.80-054236.9 & 0.23006 & 12.46 & 0.56 & 0.54 & \\
\hline 1SWASP J034439.97+030425.5 & 0.22988 & 14.25 & 0.20 & 0.17 & \\
\hline 1SWASP J161334.28-284706.7 & 0.22978 & 12.43 & 0.49 & 0.45 & \\
\hline 1SWASP J050904.45-074144.4 & 0.22958 & 13.29 & 0.64 & 0.59 & \\
\hline 1SWASP J111931.48-395048.2 & 0.22949 & 10.80 & 0.11 & 0.11 & ASAS J111932-3950.8 \\
\hline 1SWASP J004050.63+071613.9 & 0.22928 & 12.25 & 0.10 & 0.09 & \\
\hline 1SWASP J210318.76+021002.2 & 0.22859 & 13.00 & 0.05 & 0.03 & \\
\hline 1SWASP J174310.98+432709.6 & 0.22853 & 13.19 & 0.44 & 0.40 & V1067 Her \\
\hline 1SWASP J214510.25-494401.1 & 0.22816 & 14.55 & 0.17 & 0.15 & \\
\hline 1SWASP J044132.96+440613.7 & 0.22815 & 14.19 & 0.39 & 0.33 & \\
\hline 1SWASP J180947.64+490255.0 & 0.22788 & 13.89 & 0.94 & 0.72 & V1104 Her \\
\hline 1SWASP J212454.61+203030.8 & 0.22783 & 14.35 & 0.15 & 0.13 & \\
\hline 1SWASP J093012.84+533859.6 & 0.22772 & 9.53 & 0.17 & 0.15 & \\
\hline 1SWASP J114929.22-423049.0 & 0.22731 & 14.19 & 0.70 & 0.55 & \\
\hline 1SWASP J120110.98-220210.8 & 0.22717 & 14.23 & 0.13 & 0.09 & \\
\hline 1SWASP J115557.80+072010.8 & 0.22701 & 15.18 & 0.33 & 0.27 & \\
\hline 1SWASP J030749.87-365201.7 & 0.22667 & 14.87 & 0.65 & 0.62 & \\
\hline 1SWASP J003033.05+574347.6 & 0.22663 & 14.89 & 0.68 & 0.27 & \\
\hline 1SWASP J160156.04+202821.6 & 0.22653 & 14.07 & 0.70 & 0.57 & \\
\hline 1SWASP J121906.35-240056.9 & 0.22637 & 15.25 & 0.63 & 0.47 & \\
\hline 1SWASP J092756.25-391119.2 & 0.22535 & 11.77 & 0.16 & 0.15 & \\
\hline 1SWASP J212808.86+151622.0 & 0.22484 & 14.49 & 0.47 & 0.34 & \\
\hline 1SWASP J173003.21+344509.4 & 0.22372 & 13.85 & 0.30 & 0.23 & \\
\hline 1SWASP J130920.49-340919.9 & 0.22284 & 13.91 & 0.19 & 0.16 & \\
\hline 1SWASP J040615.79-425002.3 & 0.22234 & 14.14 & 0.52 & 0.50 & \\
\hline 1SWASP J173828.46+111150.2 & 0.22168 & 13.80 & 0.24 & 0.24 & \\
\hline 1SWASP J183738.17+402427.2 & 0.22131 & 14.45 & 0.27 & 0.25 & \\
\hline 1SWASP J064501.21+342154.9 & 0.22105 & 14.11 & 0.35 & 0.35 & \\
\hline 1SWASP J074658.62+224448.5 & 0.22085 & 14.06 & 0.53 & 0.46 & \\
\hline 1SWASP J055418.43+442549.8 & 0.21825 & 12.73 & 0.36 & 0.29 & \\
\hline 1SWASP J224747.20-351849.3 & 0.21822 & 13.89 & 0.20 & 0.16 & \\
\hline 1SWASP J133105.91+121538.0 & 0.21801 & 10.36 & 0.22 & 0.18 & \\
\hline 1SWASP J080150.03+471433.8 & 0.21751 & 13.40 & 0.66 & 0.64 & \\
\hline 1SWASP J041120.40-230232.3 & 0.21633 & 13.95 & 0.41 & 0.36 & \\
\hline 1SWASP J234401.81-212229.1 & 0.21367 & 11.64 & 0.11 & 0.09 & \\
\hline 1SWASP J221058.82+251123.4 & 0.21299 & 15.13 & 0.18 & 0.16 & \\
\hline 1SWASP J195900.31-252723.1 & 0.21275 & 14.64 & 0.47 & 0.43 & \\
\hline 1SWASP J022727.03+115641.7 & 0.21095 & 14.95 & 0.28 & 0.24 & \\
\hline 1SWASP J115605.88-091300.5 & 0.21091 & 12.46 & 0.05 & 0.04 & \\
\hline 1SWASP J151652.90+004835.8 & 0.21073 & 13.97 & 0.10 & 0.10 & \\
\hline 1SWASP J092328.76+435044.8 & 0.21013 & 13.03 & 0.04 & 0.03 & \\
\hline 1SWASP J142312.63-222425.1 & 0.20964 & 13.19 & 0.06 & 0.05 & \\
\hline 1SWASP J210423.76+073140.4 & 0.20908 & 13.30 & 0.05 & 0.04 & \\
\hline 1SWASP J022050.85+332047.6 & 0.19264 & 13.13 & 0.31 & 0.19 & GSC 2314-0530 \\
\hline
\end{tabular}

described earlier (Dimitrov \& Kjurkchieva 2010). The SuperWASP light curve of this latter object is shown separately in Fig. 3, folded at its confirmed period of 0.1926 d. Whilst we agree with the periods previously reported for ASAS J111932-3950.8 (0.2294 d, Parihar et al. 2009) and V1104 Her (0.2279 d, Akerlof et al. 2000), we find better periods for V1067 Her and ROTSE1 J170240.11+151122.7. The ROTSE survey had previously suggested periods of $0.2581 \mathrm{~d}$ and $1.210 \mathrm{~d}$ respectively for these two objects (Akerlof et al. 2000), but we show them to have periods of $0.2285 \mathrm{~d}$ and $0.2312 \mathrm{~d}$ respectively.

\section{Discussion}

\subsection{Previous short-period samples}

For a number of years the shortest period main sequence eclipsing binary known was the faint $(V=18.3)$ object OGLE BW03 
A. J. Norton et al.: WASP short period eclipsing binaries
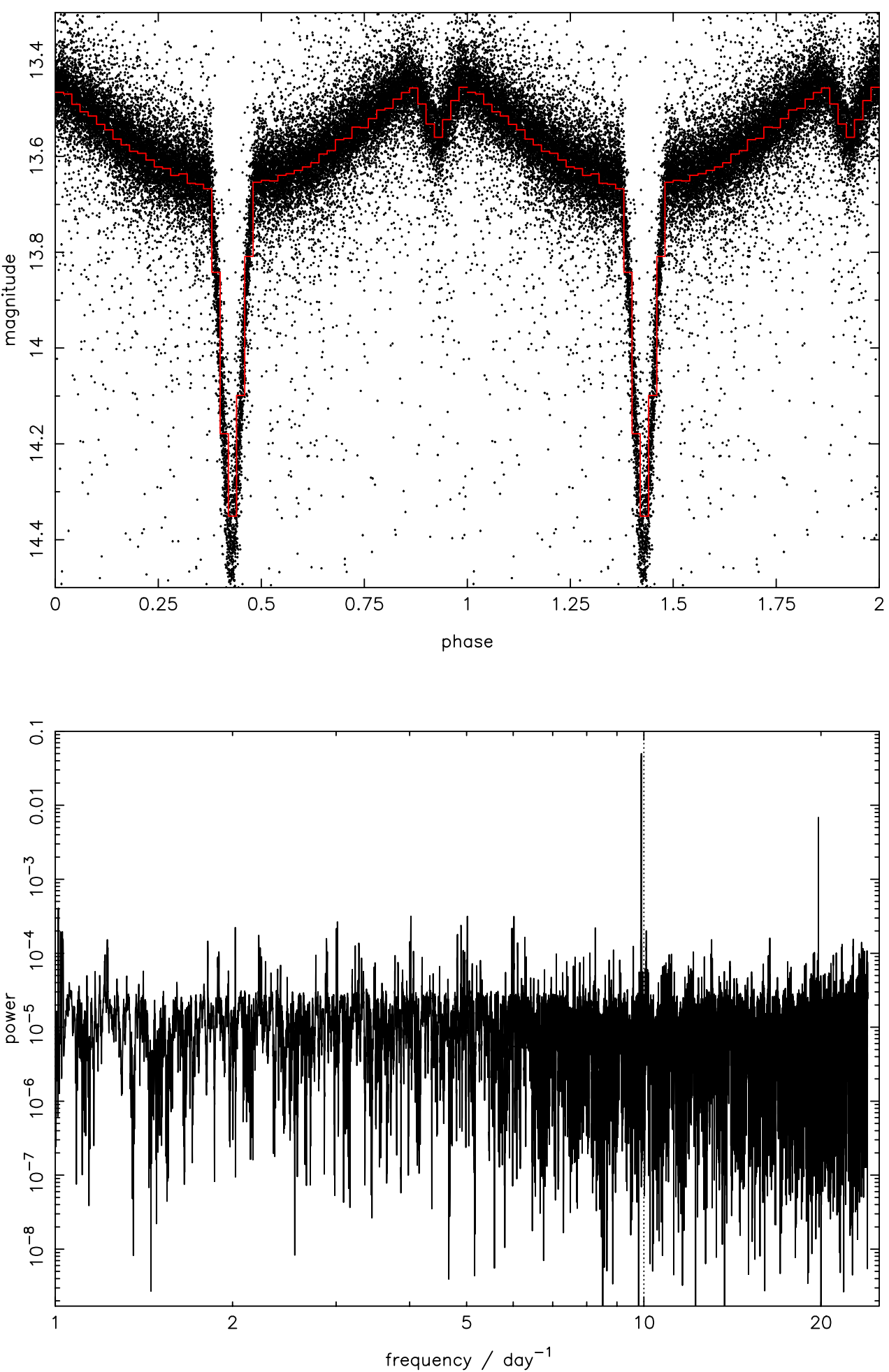

Fig. 4. (Top) The SuperWASP light curve of the sdB+dM eclipsing binary NY Vir (1SWASP J133848.16-020149.3), folded at a period of 145.463 min. Phase zero corresponds to 2004 January 1st 00:00UT. The mean folded light curve (in 50 bins) is shown by an over-plotted line. (Bottom) The associated power spectrum, with the frequency corresponding to the orbital period indicated by a dotted line.

V038, with an orbital period of 0.1984 d (Maceroni \& Rucinski 1997; Maceroni \& Montalban 2004). This was modelled as consisting of two M3 dwarfs which are almost, but not quite in contact, with masses of $0.44 M_{\odot}$ and $0.41 M_{\odot}$. This object is not present in the SuperWASP database owing to its faintness.

In presenting the short-period end of the contact binary period distribution based on the All-Sky Automated Survey
(ASAS), Rucinski (2007) found only three systems in the period range $0.200 \mathrm{~d}<P<0.225 \mathrm{~d}$ (and none at shorter periods). The only one of these they list as a confirmed W UMa system is ASAS J083128+1953.1 with a period of $0.2178 \mathrm{~d}$. Rucinski \& Pribulla (2008) subsequently presented further photometric and spectroscopic observations of this system and argued the case for this system as the shortest period field contact binary. 


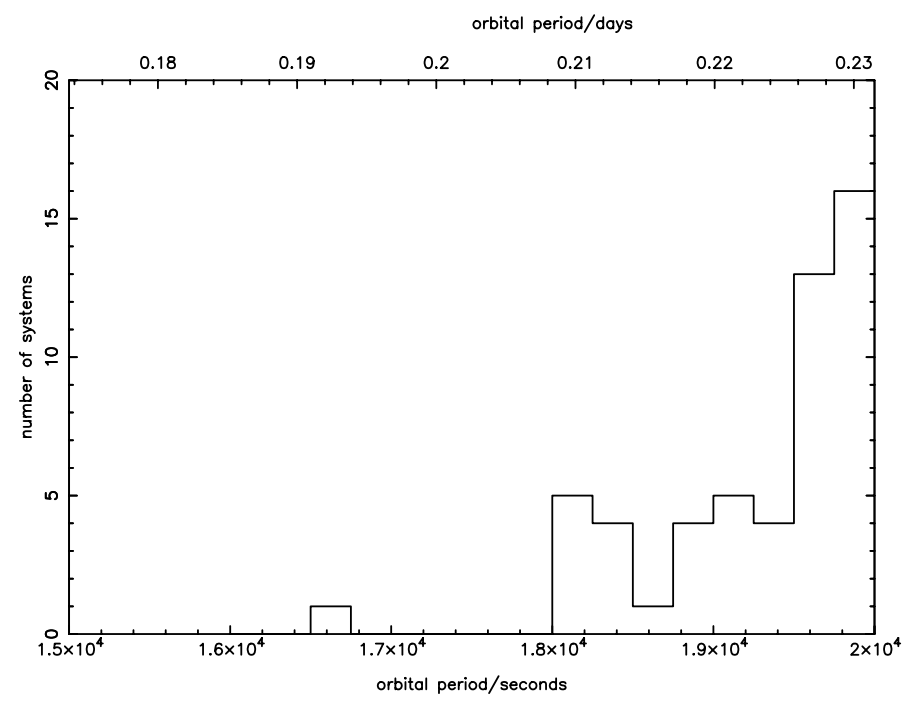

Fig. 5. The orbital period distribution of the candidate eclipsing binaries found in the range $15000 \mathrm{~s}(\sim 0.175 \mathrm{~d})$ to $20000 \mathrm{~s}(\sim 0.23 \mathrm{~d})$. The bin size is $250 \mathrm{~s}$.

This object is indeed present in the SuperWASP database (as 1SWASP J083127.87+195303.5), with the same period, but the light curve is poorly sampled (fewer than 1000 data points) and jumps between two brightness levels, presumably as a result of another star sometimes falling within the photometric aperture. It nonetheless displays a broadly sinusoidal modulation when folded at this period (see Fig. 7a). Since the modulation profile did not match that of a classic contact binary, it was not selected as a candidate eclipsing binary by the current exercise.

More recently, Pribulla et al. (2009) looked at two of the candidate short period systems listed by Rucinski (2007). They found that one of these stars (ASAS J113031-0101.9) in fact has a period longer than that originally suggested $(0.2710 \mathrm{~d}$ instead of $0.2131 \mathrm{~d}$ ). The other candidate (ASAS J071829-0336.7) was confirmed as a contact binary with a period of $0.2113 \mathrm{~d}$. As with the object discussed by Rucinksi \& Pribulla, this one too is present in the SuperWASP database (as 1SWASP J071828.67033639.5), but the light curve is very poorly sampled (fewer than 100 data points). Nonetheless, when the SuperWASP data are folded at the ASAS period, a profile characteristic of a W UMa star is revealed (see Fig. 7b). However, given the few data points, this object was not included in the sample that were examined here.

A survey of a 0.25 square degree region of the Galactic plane using the ESO-2.2 m telescope by Miller et al. (2010) yielded more than half a million light curves down to $R \sim 24.5$. Amongst this set they found 1318 variable stars, 533 of which were W UMa stars. This included seven candidate contact binaries with periods less than $0.23 \mathrm{~d}$, three of which have periods at or below the period cut-off: V-737 (0.2109 d), V-301 (0.2143 d) and $\mathrm{V}-1085(0.2199 \mathrm{~d})$. Each of these stars is relatively faint $(R$ magnitudes in the range $\sim 19-22$ ) and so they are not amenable to detailed follow-up. None of these are present in the SuperWASP database, owing to their location and faintness.

As noted in the introduction, all of the stars discussed above have recently been superseded as the shortest period eclipsing binary with main sequence components by GSC 2314-0530 (=1SWASP J022050.85+332047.6) with a period of $0.1926 \mathrm{~d}$ (Norton et al. 2007; Dimitrov \& Kjurkchieva 2010), as shown in Fig. 3 of the present paper.
As a result of our work, however, Fig. 5 and Table 1 show that we can now add a further 22 candidates to the set of eclipsing binaries with the shortest periods $(0.200-0.225$ d). Four of these are brighter than magnitude 13 , so might have been accessible to ASAS. In addition we find a further 30 candidates in the period range at which the sharp cut-off occurs $(0.225-0.230 \mathrm{~d})$, although four of this latter set were already known, two of them had the wrong period previously recorded. Rucinski (2007) commented at the time of the ASAS work that the statistics of the sample at such short periods were very limited, despite the fact that ASAS covered around 3/4 of the sky and extended down to magnitude $\sim 13$. SuperWASP covers a similar fraction of the sky (avoiding the Galactic plane) but is sensitive down to magnitude $\sim 15$, i.e. over six times fainter, and as a result increases the sample by a factor of more than six.

\subsection{Parameter relationships}

Deb \& Singh (2010) have recently presented an analysis of the light curves of 62 binary stars (mostly contact binaries) from the ASAS-3 survey. In particular they show that there is a rather tight correlation between the period and $(J-K)$ colour of the contact binaries in their sample (see their Fig. 11). The relationship is parameterized by

$(J-K)=(0.11 \pm 0.01) P^{-1.19 \pm 0.08}$

where $P$ is in days. Although their relationship is well constrained, the majority of their sample have periods around $0.4 \mathrm{~d}$ and they include relatively few objects close to the period cutoff. In Fig. 8 we show the 2MASS $(J-K)$ colours of our sample, against period, with Eq. (1) over-plotted. Although the fit is reasonably good, there is significant scatter in the colours of these short period systems.

Gazeas \& Stepien (2008) and Gazeas \& Niarchos (2006) demonstrated that there are clear correlations between the masses of the components of contact binaries and their orbital periods, and also between the radii of the components and their orbital periods. In particular, the period cut-off of around $0.22 \mathrm{~d}$ corresponds to primary and secondary masses of around $0.85 \mathrm{M}_{\odot}$ and $0.3 M_{\odot}$ and radii of around $0.7 M_{\odot}$ and $0.5 M_{\odot}$ respectively. These masses and radii correspond to stars of spectral type $\mathrm{K}$ and thus match the observed $V-K$ colours we see in our sample (Fig. 6). The mass and radius estimates of Gazeas \& Stepien (2008) are good to around $15 \%$ accuracy. However, the short period end of these correlations is defined by only three stars: CC Com (0.2211 d), V523 Cas (0.2337 d) and RW Com $(0.2373 \mathrm{~d})$, and there is considerable scatter between their parameters. Increasing the number of objects in this period range which can be modelled will therefore allow these correlations to be tested further and improved upon.

Another interesting result is that of Qian (2001) who demonstrates a possible relation between the period change and the mass ratio of contact binaries. The systems he studies are all at longer periods than those discussed here, so it would be interesting to look for possible period changes in these more compact systems to see if the effect is present here also. In fact, a few of the objects in our samples may already display evidence for period changes. The folded light curves of 1SWASP J150822.80 054236.9 and 1SWASP J183738.17+402427.2 for instance, show a broadening of their profiles which cannot be reduced by refining the period further. This is likely to indicate that the period is changing slightly over the several year baseline of the SuperWASP observations. 
A. J. Norton et al.: WASP short period eclipsing binaries

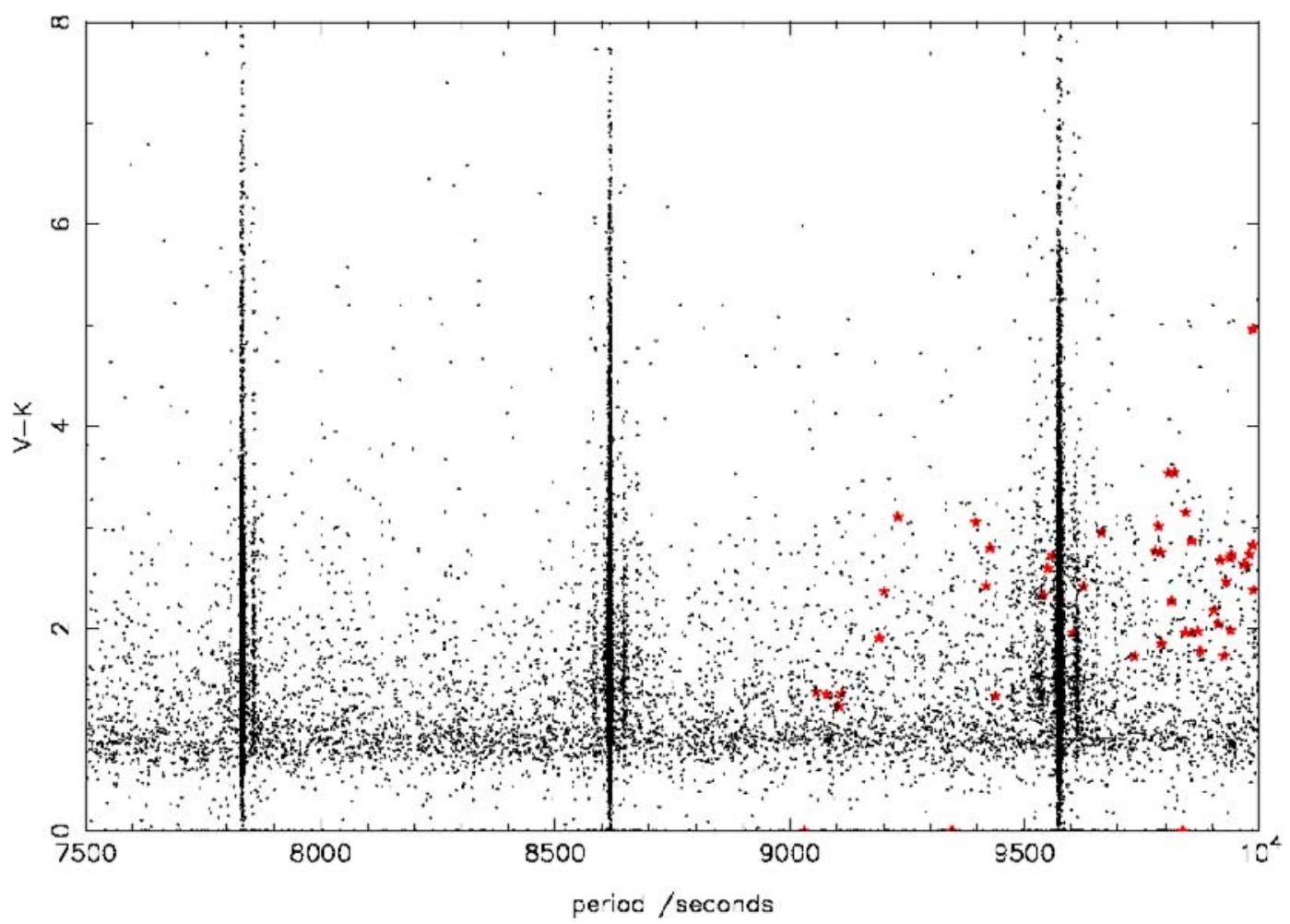

Fig. 6. The $V-K$ colours of the variable stars found in the period range $7500 \mathrm{~s}$ to $10000 \mathrm{~s}$. The candidate W UMa stars from Table 1 are shown as filled, larger symbols at half their proposed orbital period in each case. All are redder than the average colours of the rest of the sample. The structures seen amongst the other points are due to systematic noise giving rise to false periods close to $1 / 9,1 / 10$ and $1 / 11$ of a sidereal day, with weaker structures at $1 / 9,1 / 10$ and $1 / 11$ of a solar day.
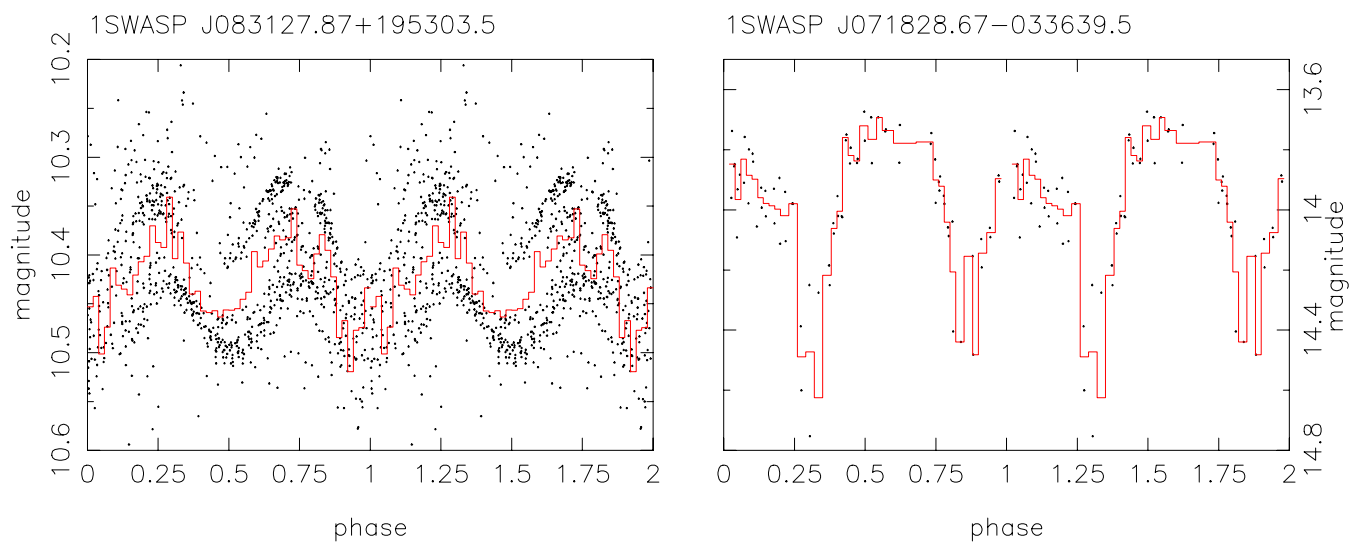

Fig. 7. Two previously identified short period contact binaries which were not picked out by the current exercise owing to their relatively poorly sampled SuperWASP light curves. a) The SuperWASP light curve of 1SWASP J083127.87+195303.5 folded at its orbital period of 0.2178 d. b) The SuperWASP light curve of 1SWASP J071828.67-033639.5 folded at its orbital period of $0.2113 \mathrm{~d}$.

\subsection{The evolution of low mass binaries}

Low mass binaries are born with a certain minimum period on the main sequence but angular momentum losses, driven by stellar winds, cause such binaries to evolve to even shorter periods as they age. Stars in such systems will expand as their core hydrogen is depleted and reach contact with their Roche lobes within a few billion years, when the orbital period has reduced to around $0.4 \mathrm{~d}$ (Stepien 2006). Detailed calculations by Stepien (2006) show that the time needed to reach a stage of Roche lobe overflow (RLOF), and hence appear as a contact binary, is around 7.5 Gyr for a primary star of $1 M_{\odot}$. However, because the angular momentum loss timescale increases substantially with decreasing stellar mass, the time to reach RLOF for a system with a primary star of mass $0.7 M_{\odot}$ increases to greater than the age of the Universe. As a result, Stepien (2006) showed that the short period limit of $\sim 0.22 \mathrm{~d}$ for contact binaries in the Galactic disc corresponds to a lower limit of around 1.1-1.2 $M_{\odot}$ for the total mass of the system (slightly less massive for systems in globular clusters). The short period cut-off for contact binaries is therefore suggested to be due to the finite age of the Galaxy - lower mass stars which could evolve to shorter periods have not yet had enough time to do so.

Stepien (2006) also notes some limitations of his analysis. In particular, he only performs calculations for initial mass ratios of 0.5 and 1.0 and assumes that the total angular momentum of the binary is approximated by the orbital angular momentum only, which rules out systems with extreme mass ratios. Stepien 


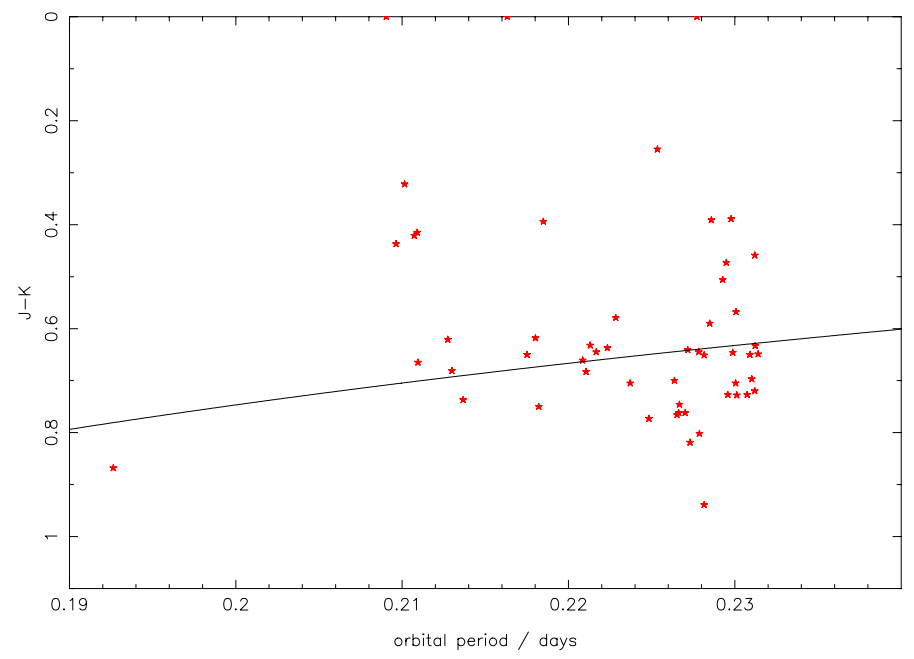

Fig. 8. The $J-K$ colours of the stars in our sample plotted against orbital period. Over-plotted is the period-colour relationship derived by Deb \& Singh (2010) for mostly longer period objects, shown in Eq. (1).

also notes that if a binary happens to lose a significant fraction of its angular momentum by other mechanisms (such as interactions with a third body, collisions within a dense stellar environment, or enhanced angular momentum loss during the pre-main sequence phase), then the orbital period during the RLOF phase may be as small as $0.15-0.20 \mathrm{~d}$. This may explain the existence of a system such as GSC 2314-0530, with a period of $0.1926 \mathrm{~d}$, which Dimitrov \& Kjurkchieva (2010) modelled as comprising stars of mass $0.51 M_{\odot}$ and $0.26 M_{\odot}$.

\section{Conclusions}

Prior to our work only a handful of W UMa stars at or below the short period cut-off were known. As a result of the candidates presented here, we now have 22 candidates in the period range considered to be below the cut-off (i.e. $0.200-0.225 \mathrm{~d}$ ) and a further 30 candidates in the period range of the cut-off itself $(0.225-0.230 \mathrm{~d})$. Along with the intriguing very short period system GSC 2314-0530, there are now well over 50 systems which can be investigated to test the ideas put forward by Stepien (2006) concerning the evolution of low mass binary stars and the mass-radius-period relationships outlined by Gazeas \& Stepien (2008). We urge others to carry out multi-colour photometry and radial velocity spectroscopic follow-up of these systems to confirm their nature.

Acknowledgements. The WASP project is funded and operated by Queen's University Belfast, the Universities of Keele, St. Andrews and Leicester, the Open University, the Isaac Newton Group, the Instituto de Astrofisica de Canarias, the South African Astronomical Observatory and by STFC.

This research has made extensive use of the SIMBAD database, operated at CDS, Strasbourg, France. We thank Harry Lehto for use of his implementation of the 1D CLEAN algorithm.

\section{References}

Akerlof, C., Amrose, S., Balsano, R., et al. 2000, AJ, 119, 1901 Butters, O. W., West, R. G., Anderson, D. R., et al. 2010, A\&A, 520, L10

Deb, S., \& Singh, H. P. 2011, MNRAS, in press [arXiv: 1011.2574] Dimitrov, D. P., \& Kjurkchieva, D. P. 2010, MNRAS, 406, 2559

Gazeas, K. D., \& Niarchos, P. G. 2006, MNRAS, 370, L29

Gazeas, K., \& Stepien, K. 2008, MNRAS, 390, 1577

Lehto, H. J. 1997, in Applications of Time Series Analysis in Astronomy and Meteorology, ed. T. Subba Rao, M. B. Priestley, \& O. Lessi (London: Chapman and Hall)

Maceroni, C., \& Rucinksi, S. M. 1997, PASP, 109, 782

Maceroni, C., \& Montalban, J. 2004, A\&A, 426, 577

Miller, V. R., Albrow, M. D., Afonso, C., \& Henning, Th. 2010, A\&A, 519, A12

Norton, A. J., Wheatley, P. J., West, R. G., et al. 2007, A\&A, 467, 785

Parihar, P., Messina, S., Bama, P., et al. 2009, MNRAS, 395, 593

Pollacco, D. L., Skillen, I., Cameron, A. C., et al. 2006, PASP, 118, 1407

Pribulla, T., Vanko, M., \& Hambalek, L. 2009, IBVS, 5886

Qian, S. 2001, MNRAS, 328, 635

Rucinski, S. M. 1992, AJ, 103, 960

Rucinski, S. M. 2007, MNRAS, 382, 393

Rucinski, S. M., \& Pribulla, T. 2008, MNRAS, 388, 1831

Stepien, K. 2006, Acta Astron., 56, 347

Tamuz, O., Mazeh, T., \& Zucker, S. 2005, MNRAS, 356, 1466 
A. J. Norton et al.: WASP short period eclipsing binaries
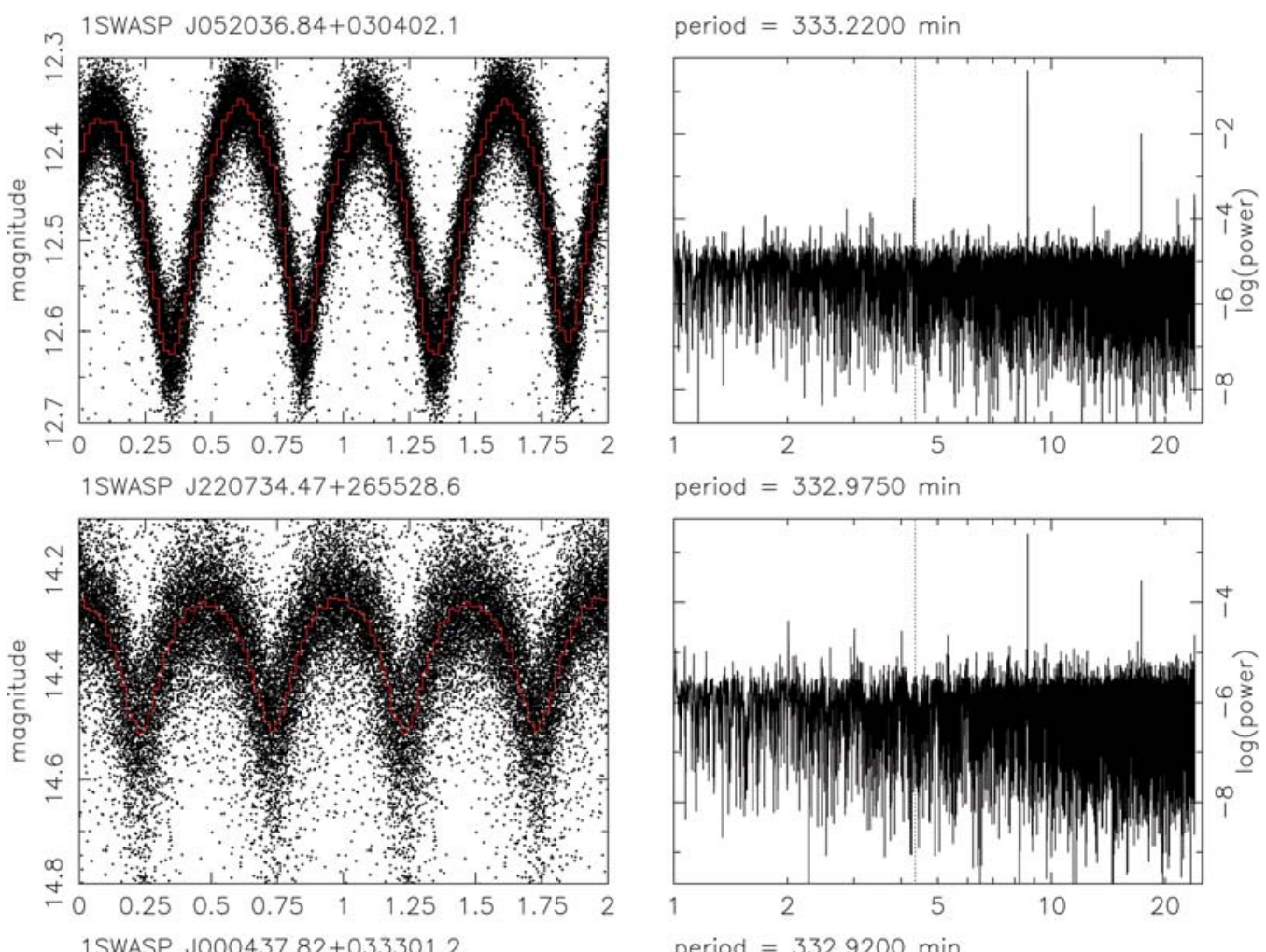

period $=332.9750 \mathrm{~min}$
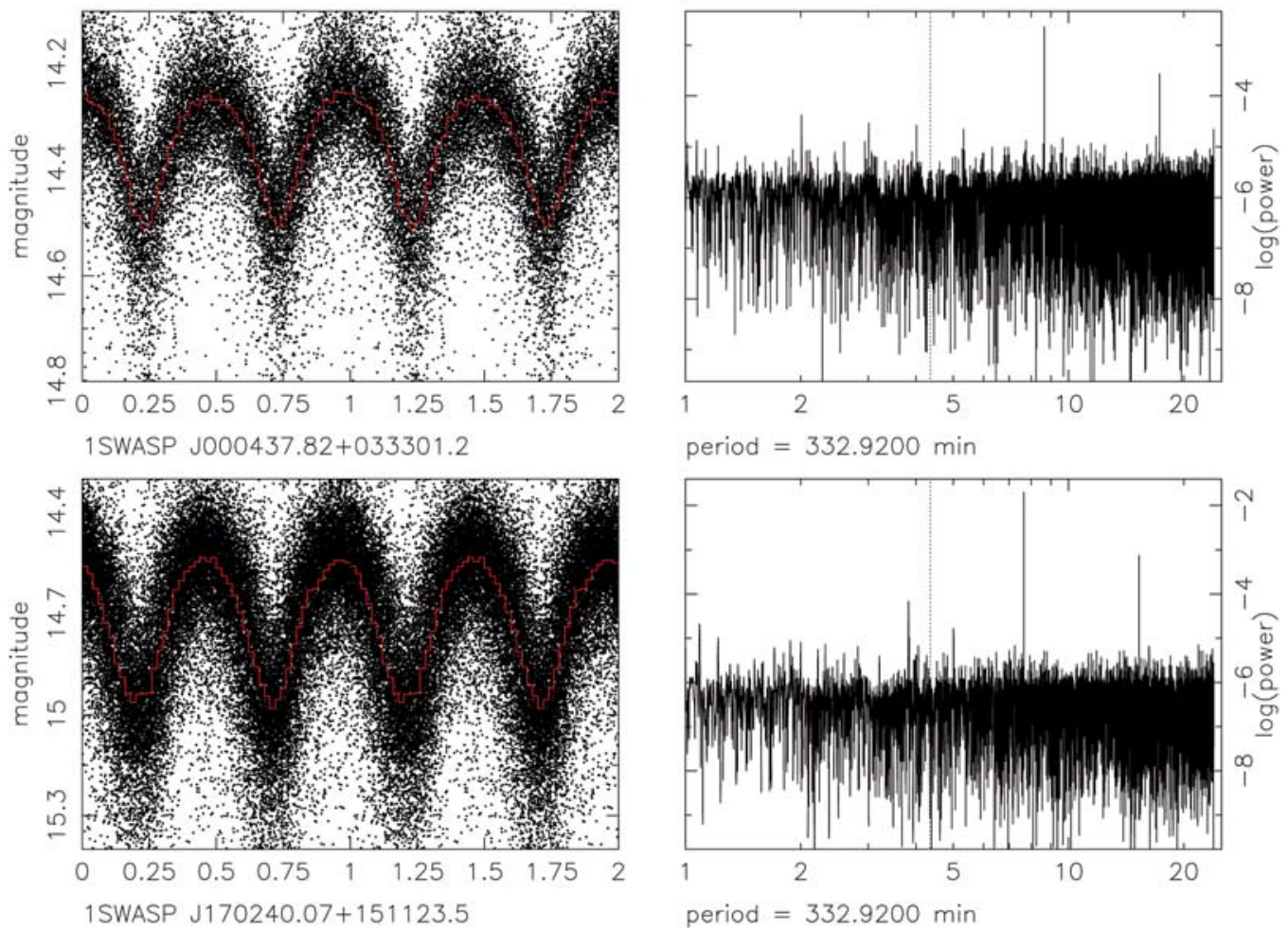

period $=332.9200 \mathrm{~min}$

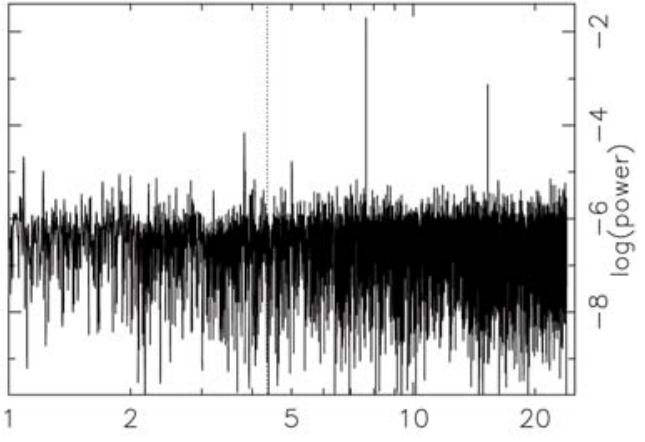

period $=332.9200 \mathrm{~min}$
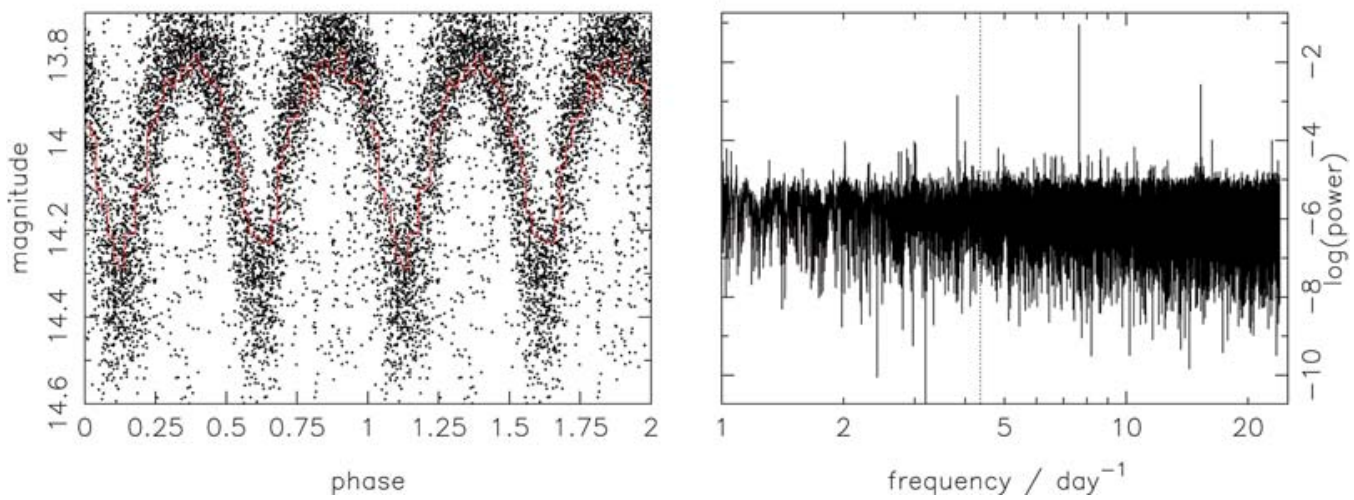

Fig. 2. (Left) SuperWASP light curves folded at the orbital period, in order of decreasing period. Phase zero corresponds to 2004 January 1 st 00:00UT in each case. The mean folded light curve (in 50 bins) is shown by an over-plotted line. (Right) The associated power spectra, with the frequency corresponding to the presumed orbital period indicated by a dotted line. The SuperWASP identifier and the presumed orbital period are written above each pair of panels. 

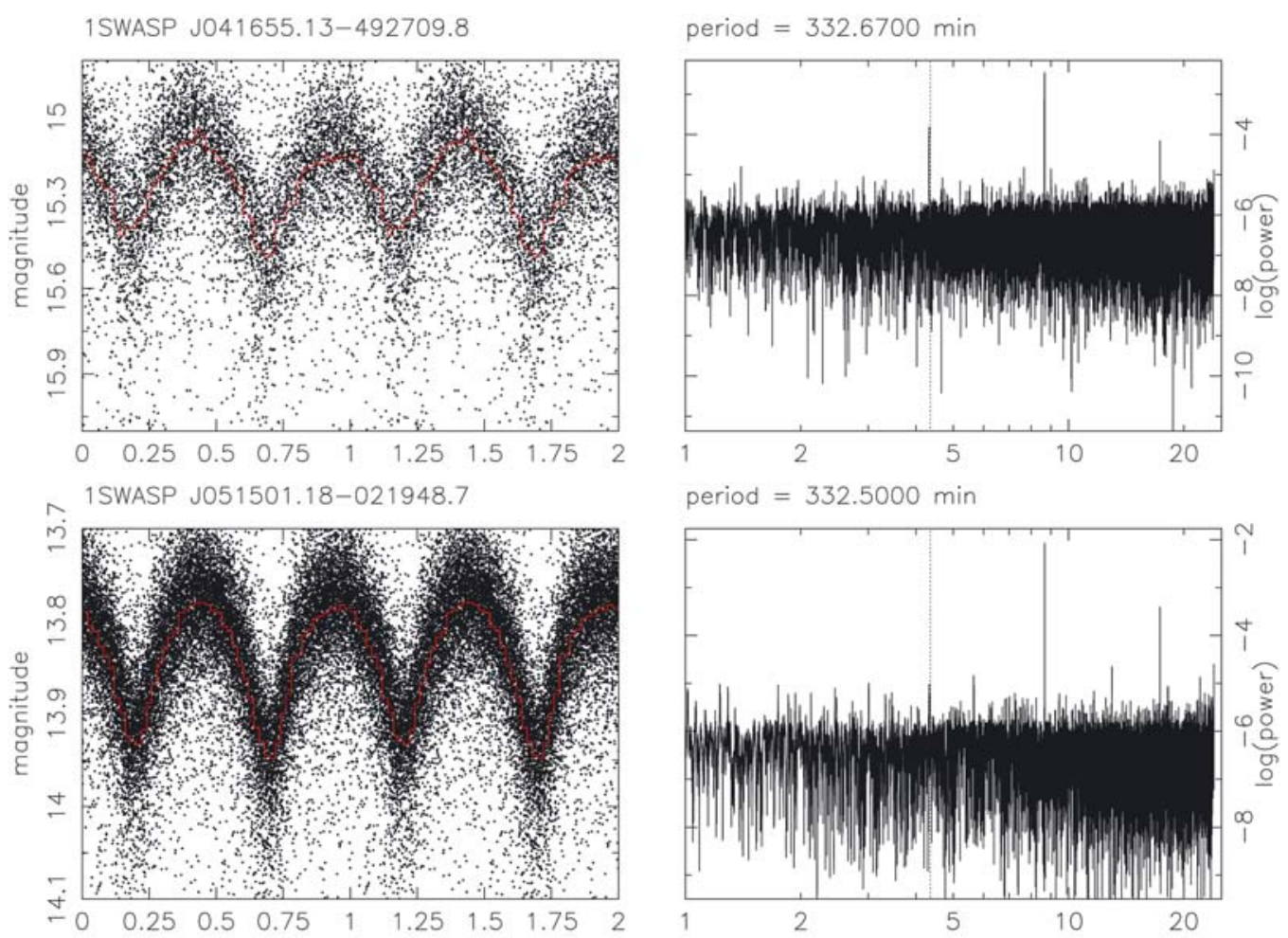

period $=332.5000 \mathrm{~min}$
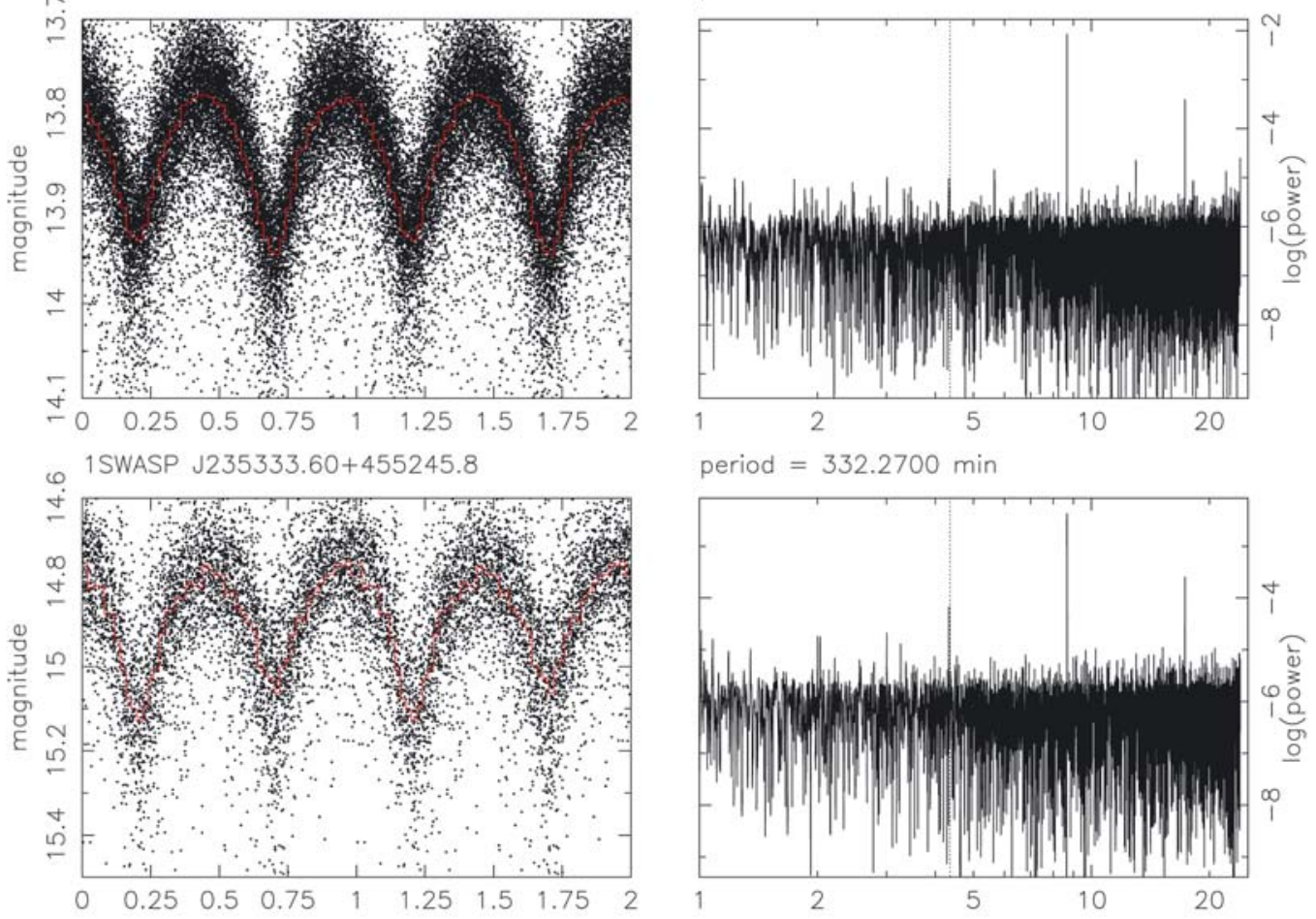

period $=332.2700 \mathrm{~min}$
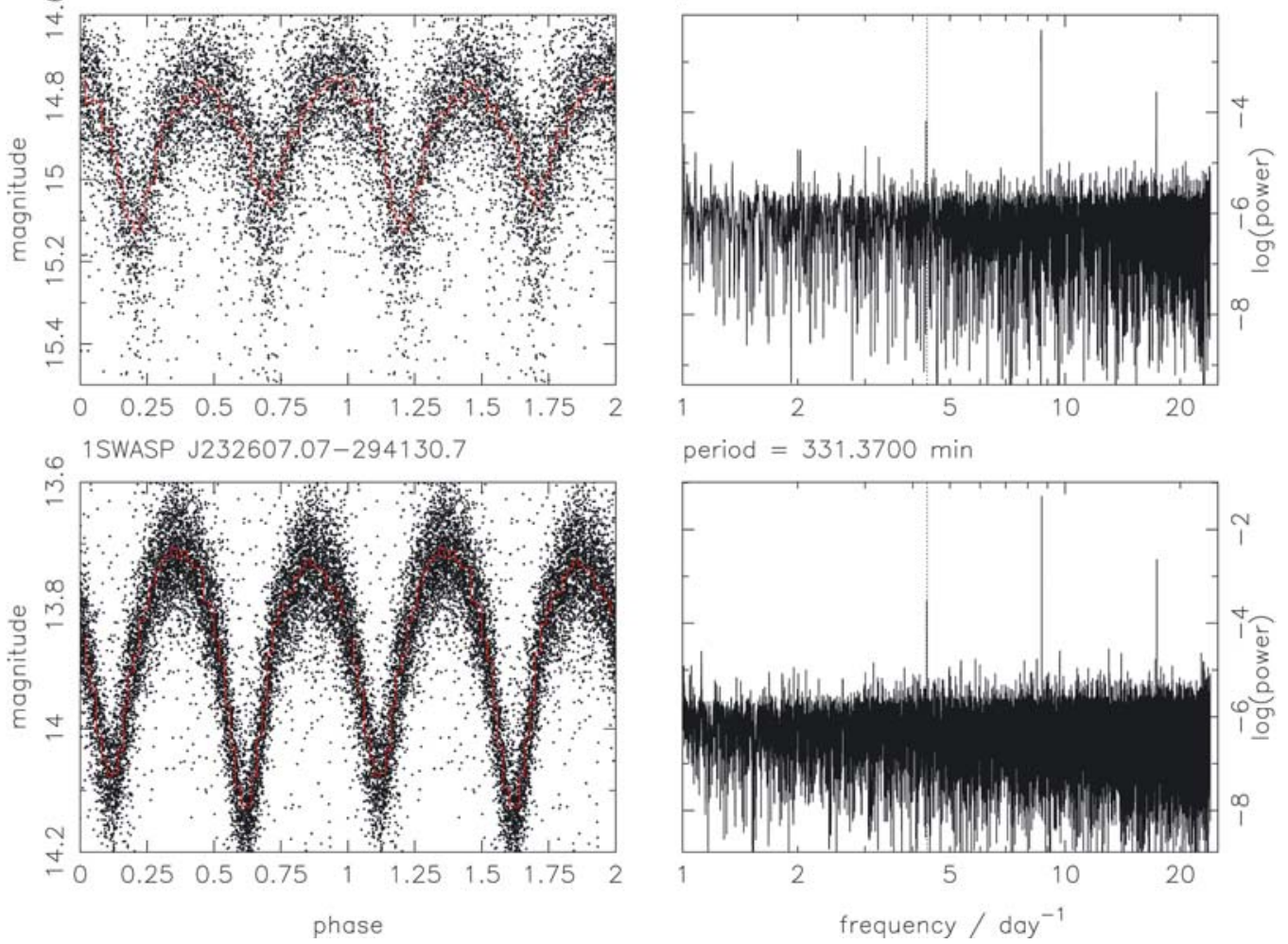

period $=331.3700 \mathrm{~min}$

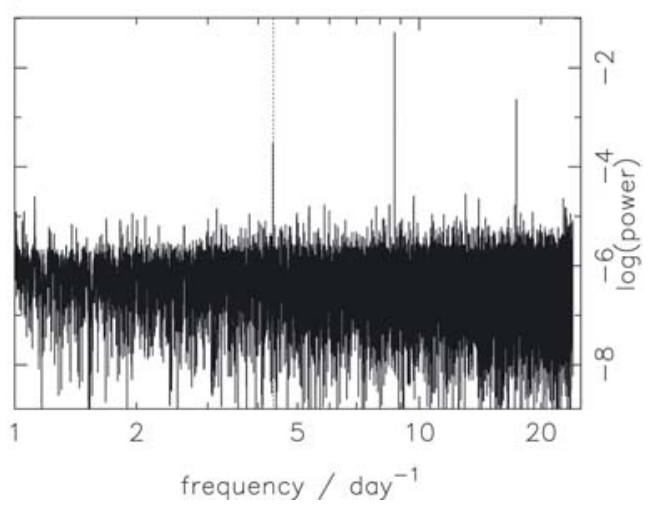

Fig. 2. continued. 
A. J. Norton et al.: WASP short period eclipsing binaries
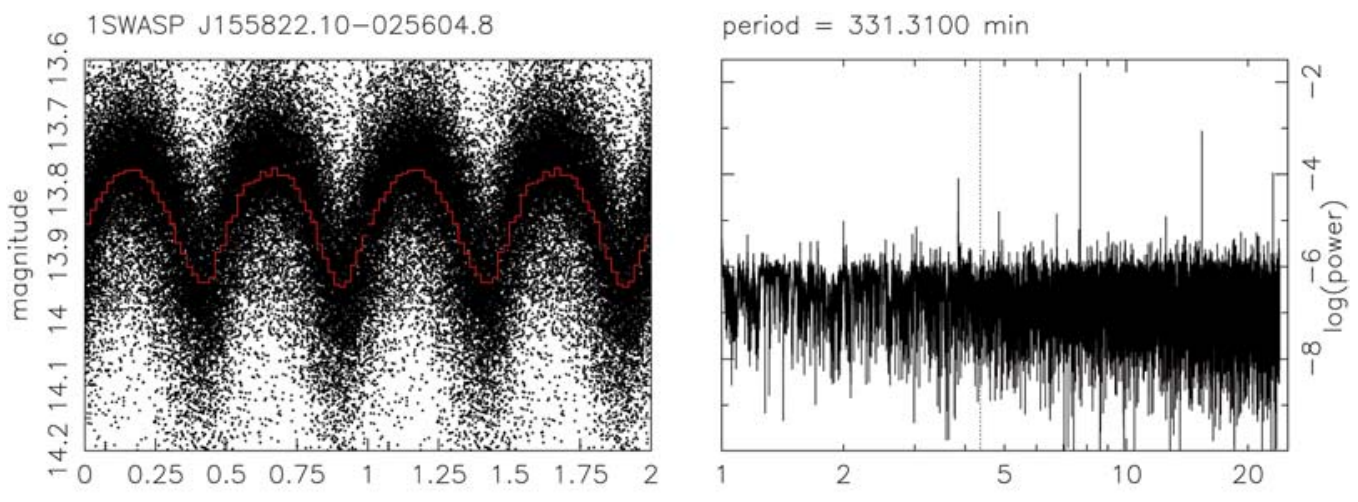

1SWASP J150822.80-054236.9

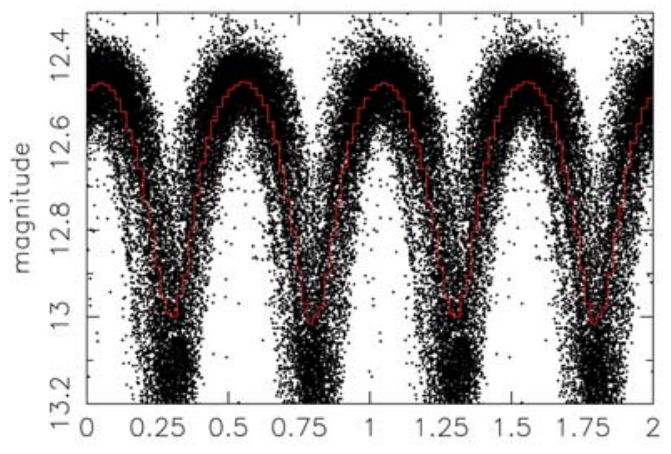

period $=331.2900 \mathrm{~min}$
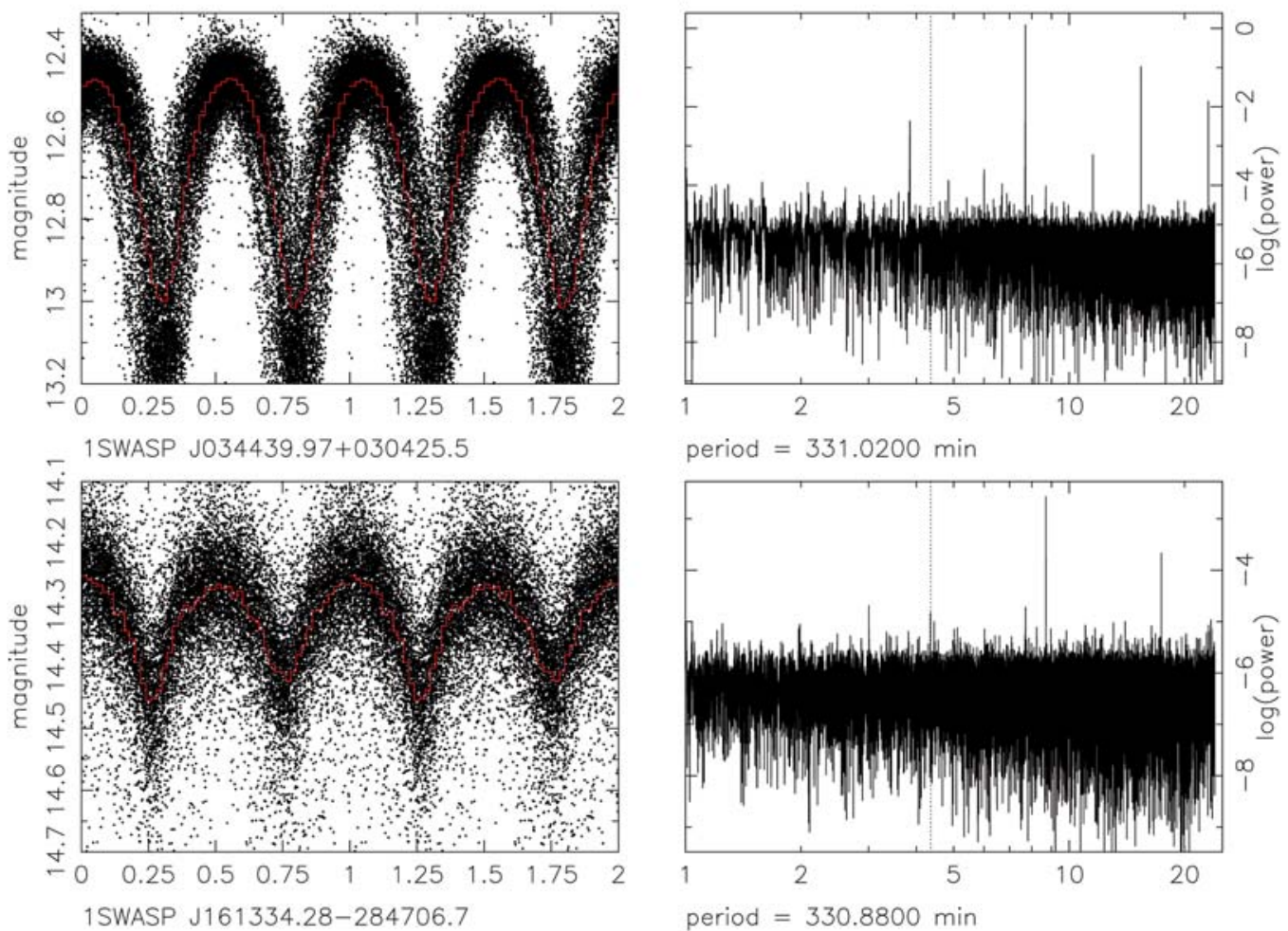

period $=331.0200 \mathrm{~min}$
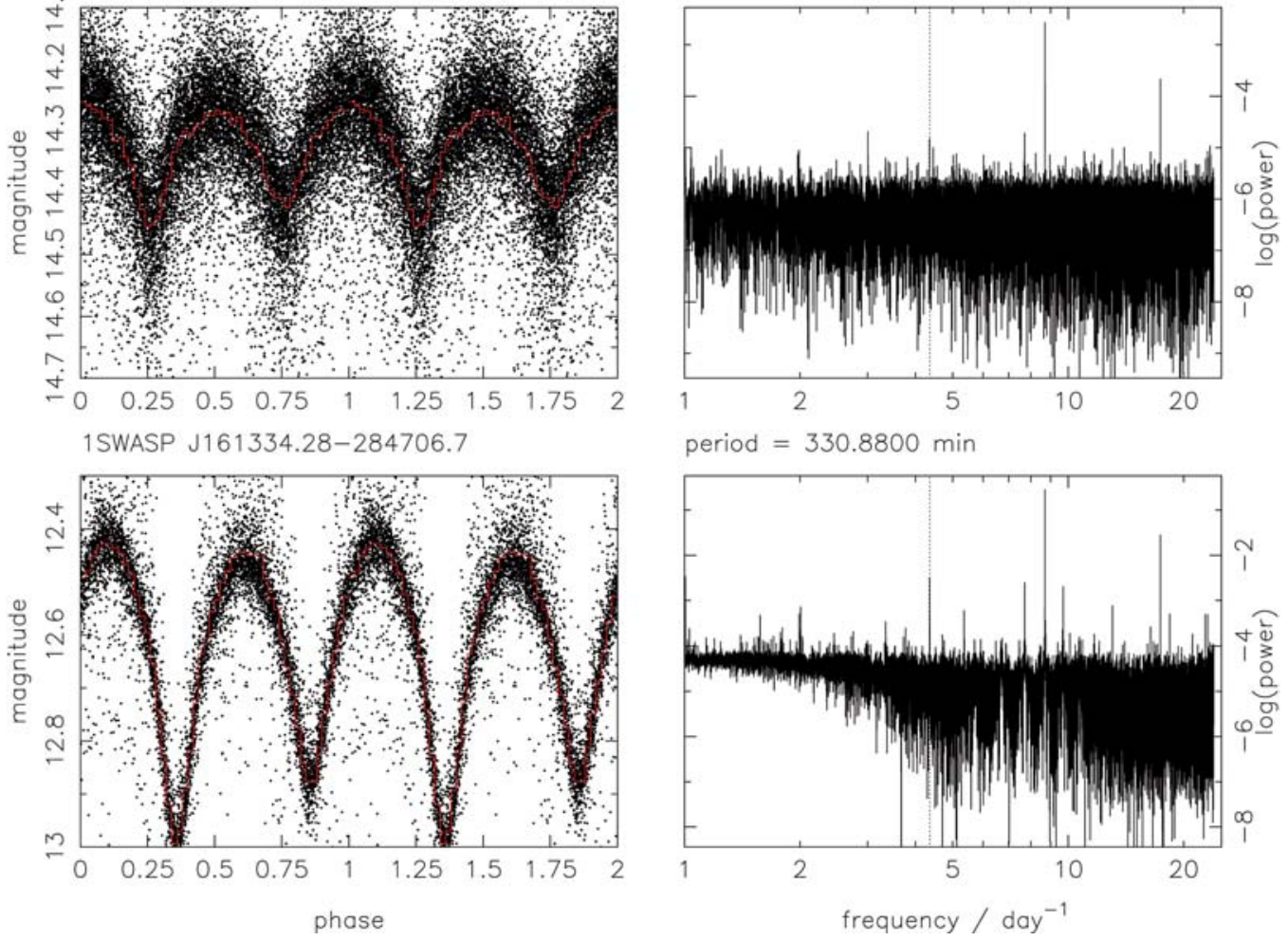

Fig. 2. continued. 

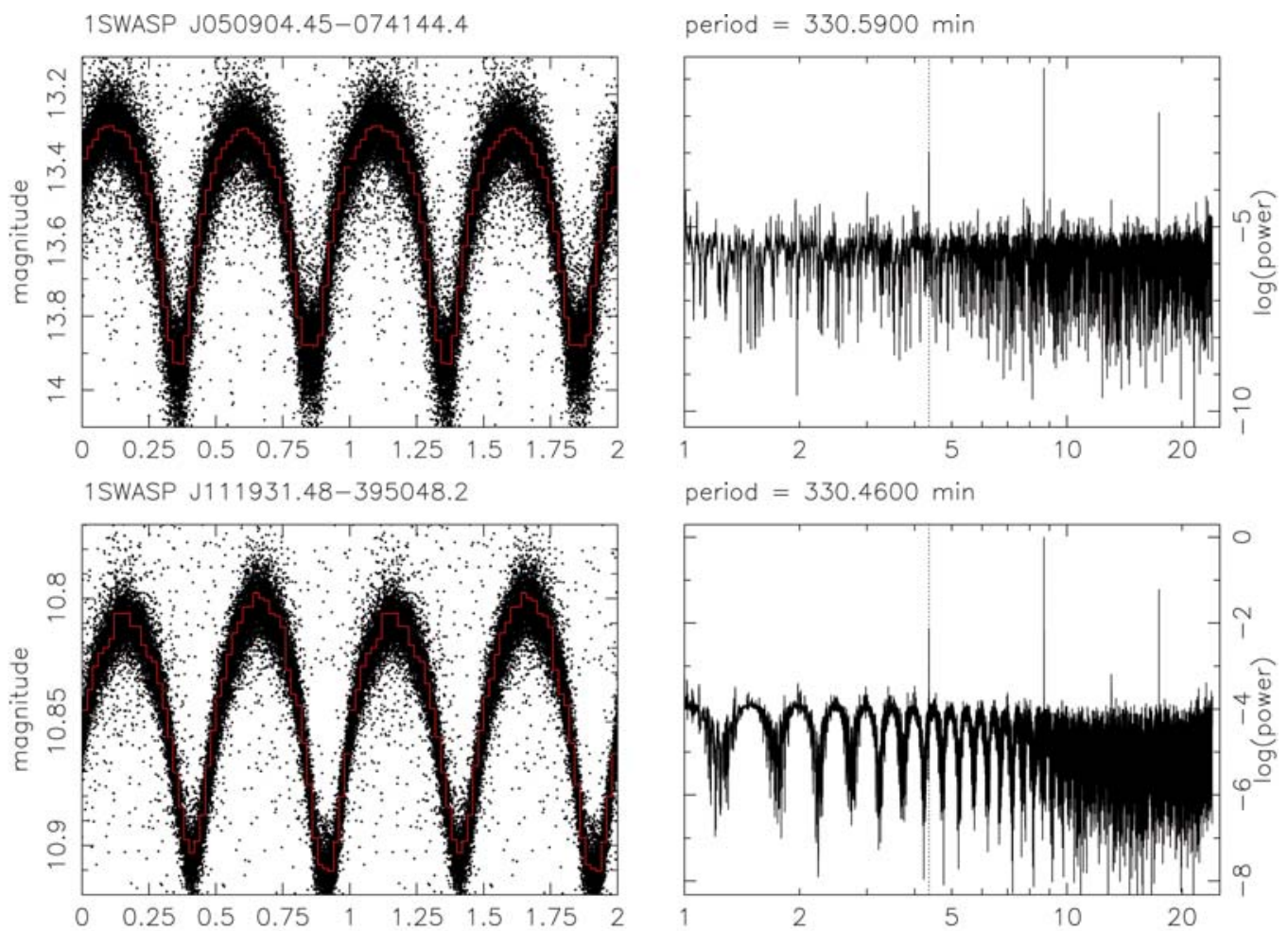

period $=330.4600 \mathrm{~min}$
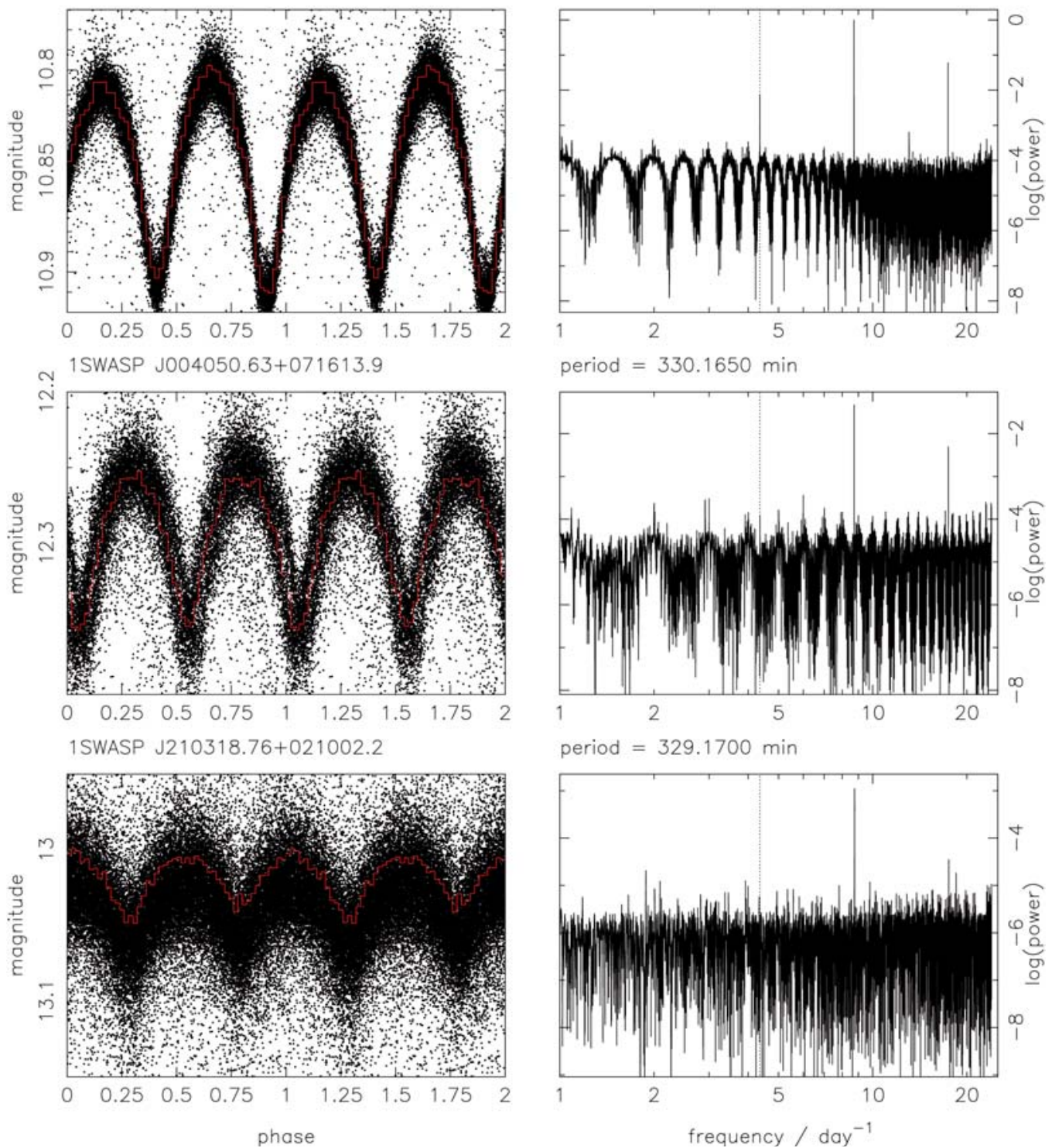

period $=329.1700 \mathrm{~min}$

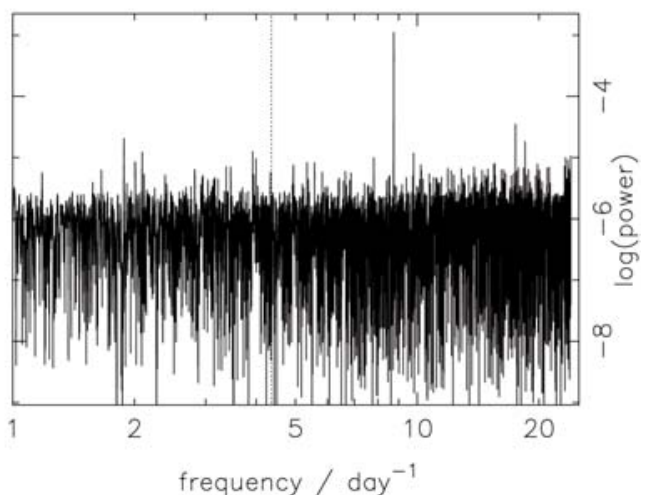

Fig. 2. continued 
A. J. Norton et al.: WASP short period eclipsing binaries
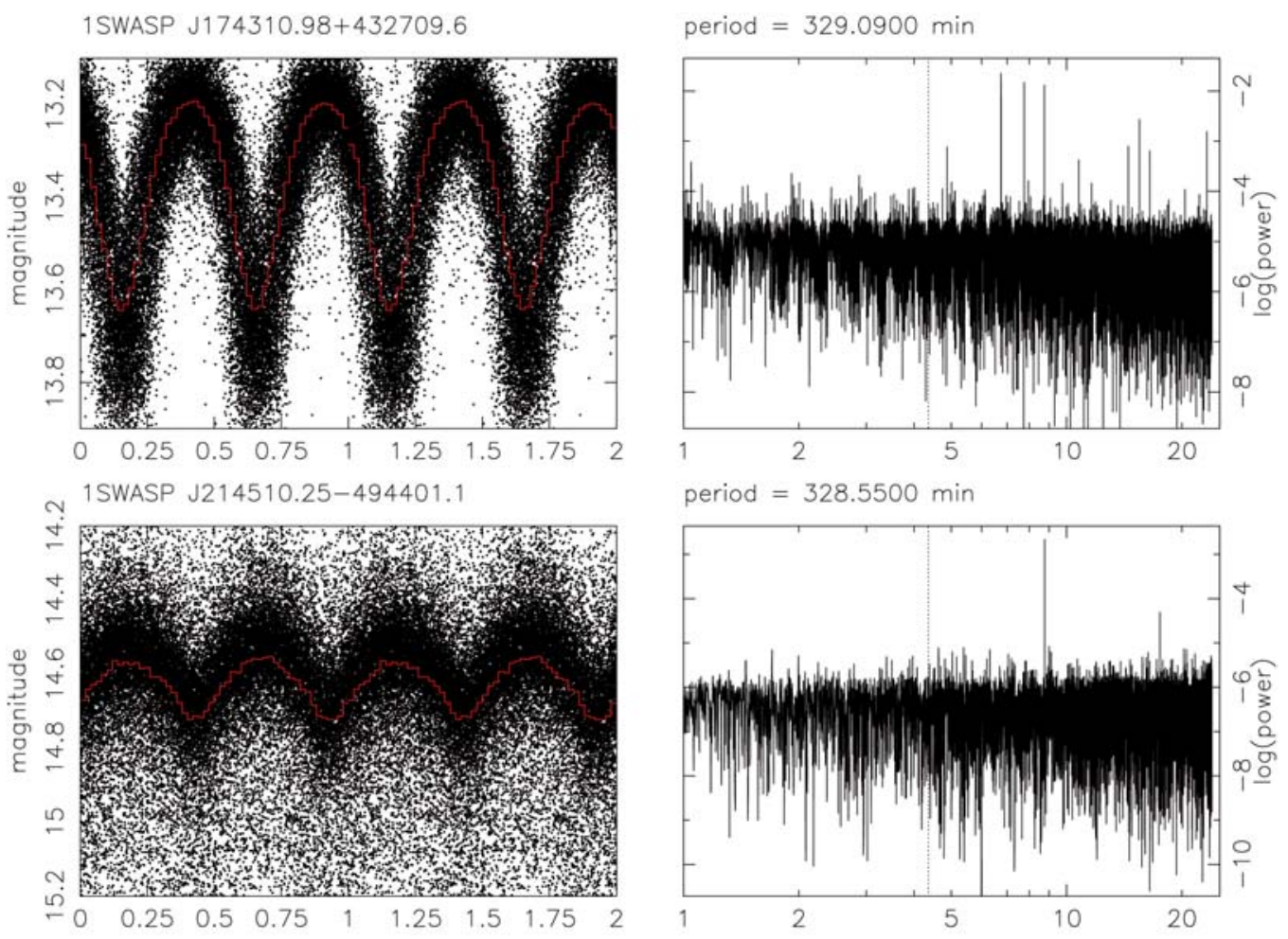

period $=328.5500 \mathrm{~min}$
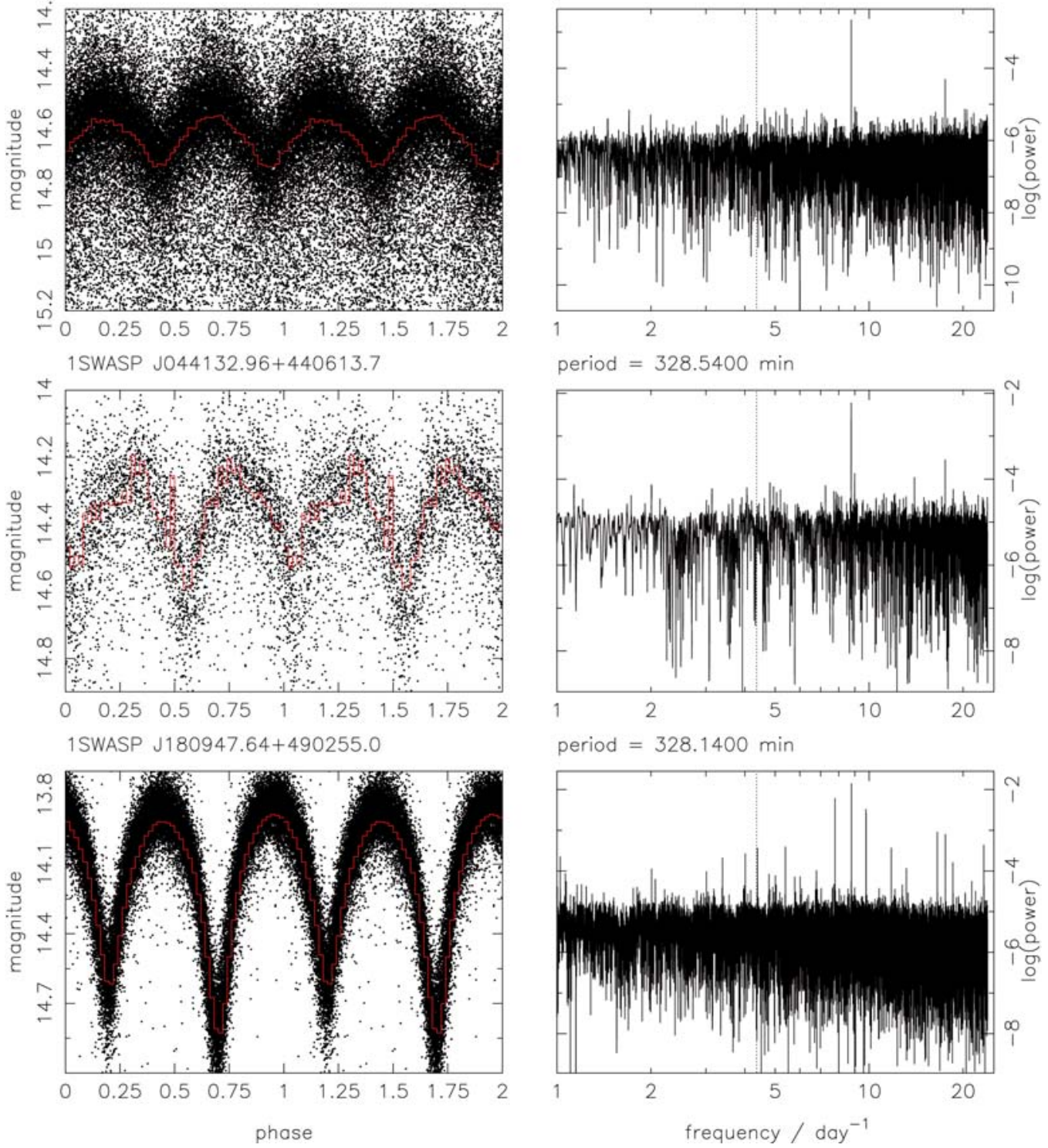

period $=328.1400 \mathrm{~min}$

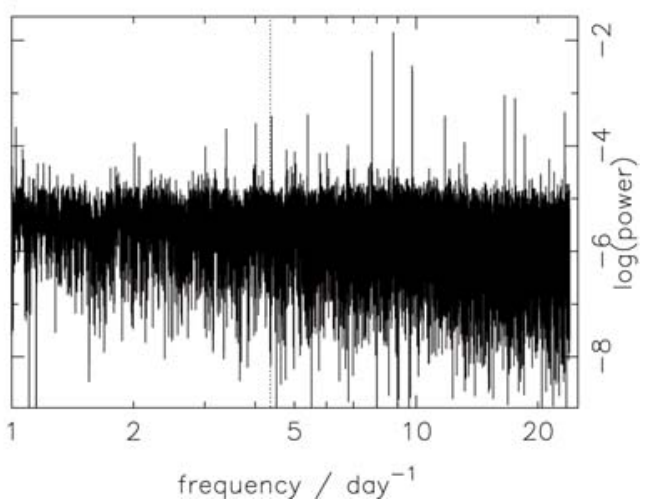

Fig. 2. continued. 

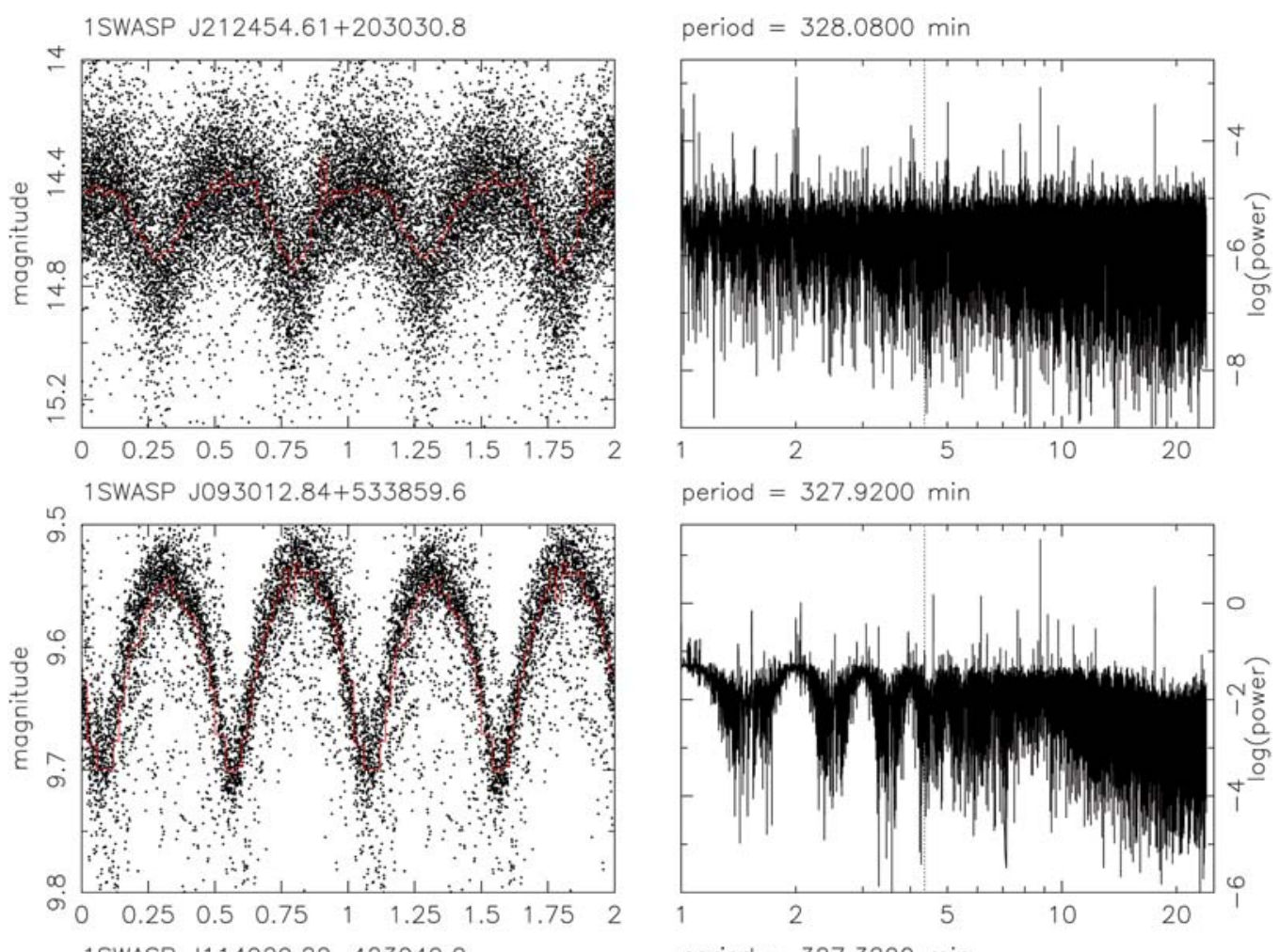

period $=327.9200 \mathrm{~min}$
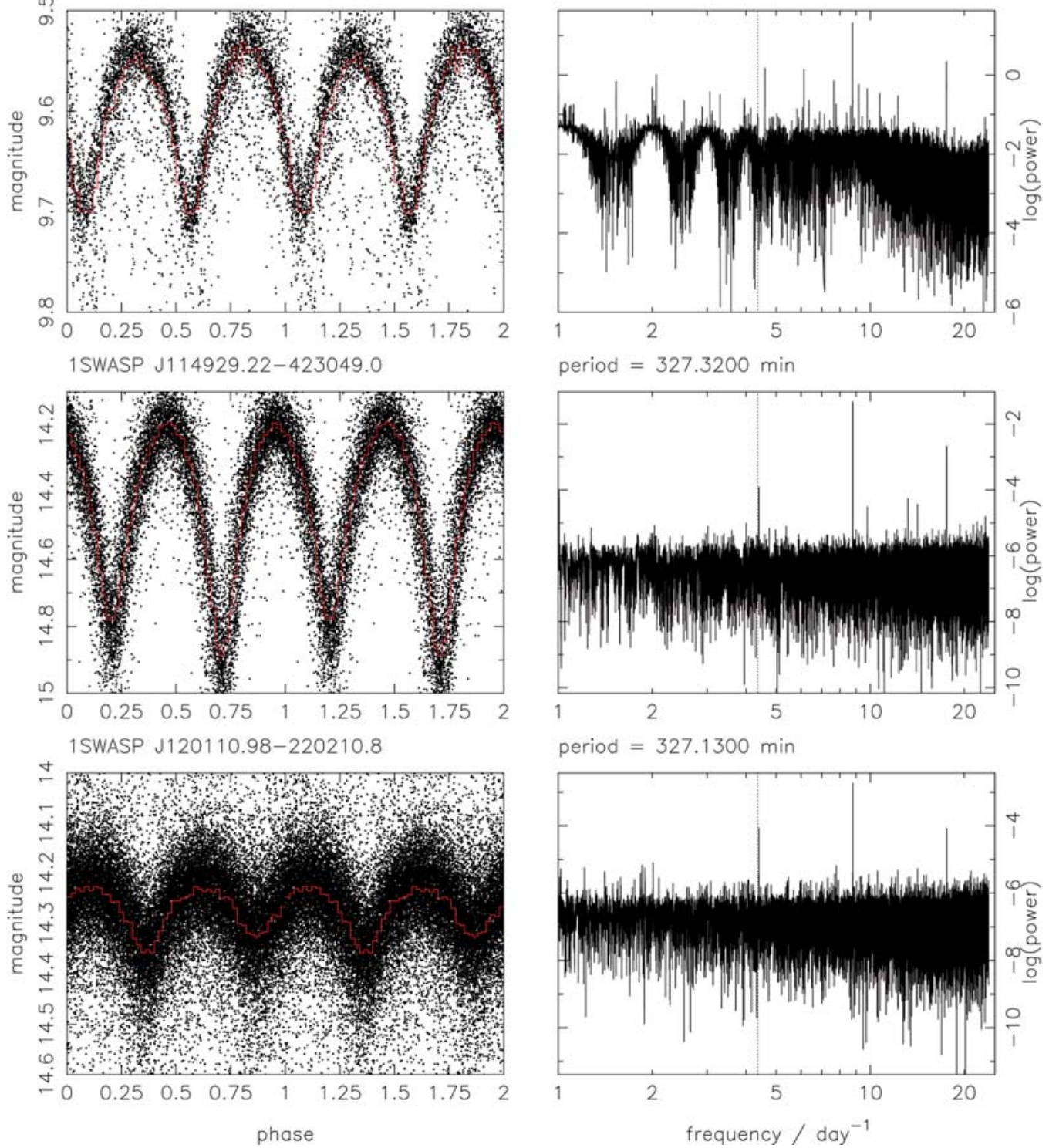

period $=327.1300 \mathrm{~min}$

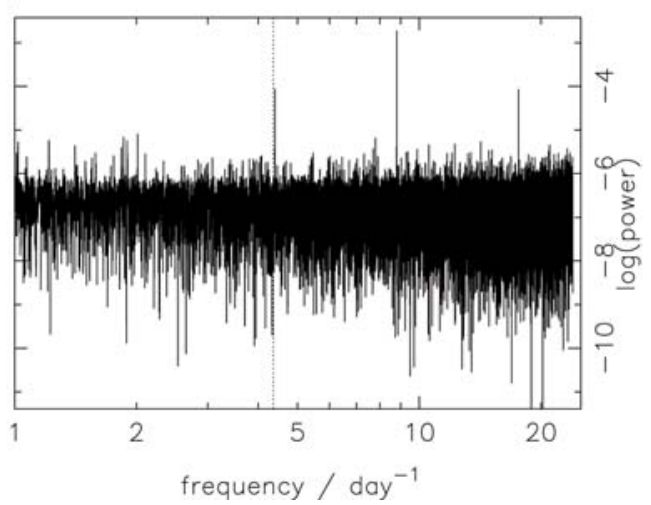

Fig. 2. continued. 
A. J. Norton et al.: WASP short period eclipsing binaries
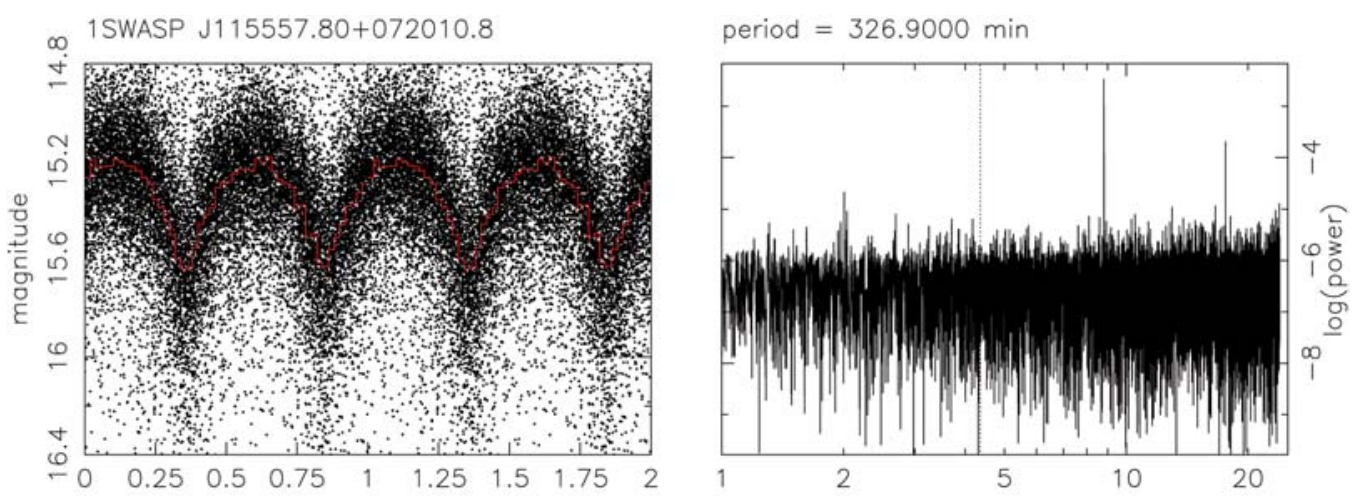

1SWASP J030749.87-365201.7

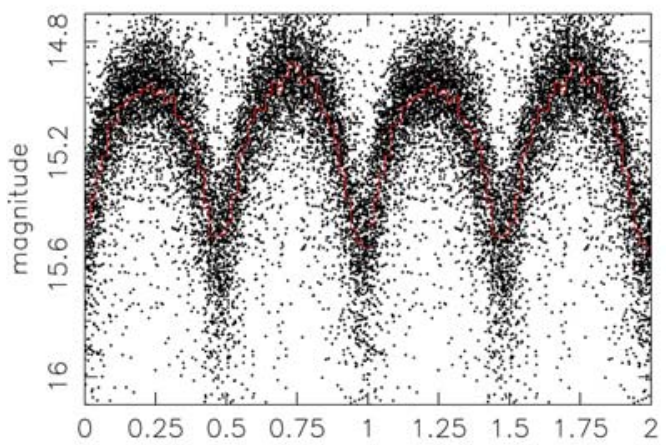

period $=326.4000 \mathrm{~min}$

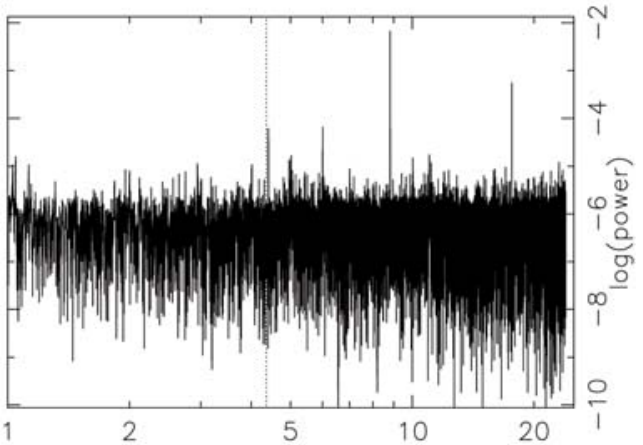

1SWASP J003033.05+574347.6

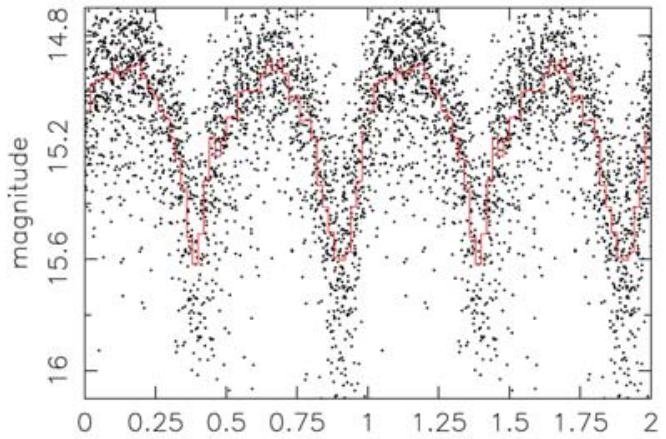

period $=326.3400 \mathrm{~min}$

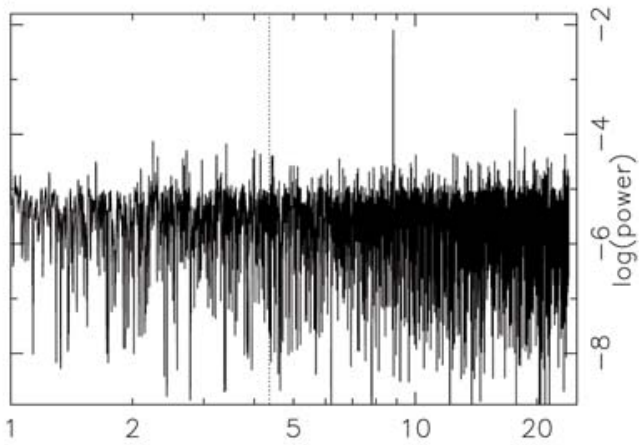

period $=326.2000 \mathrm{~min}$
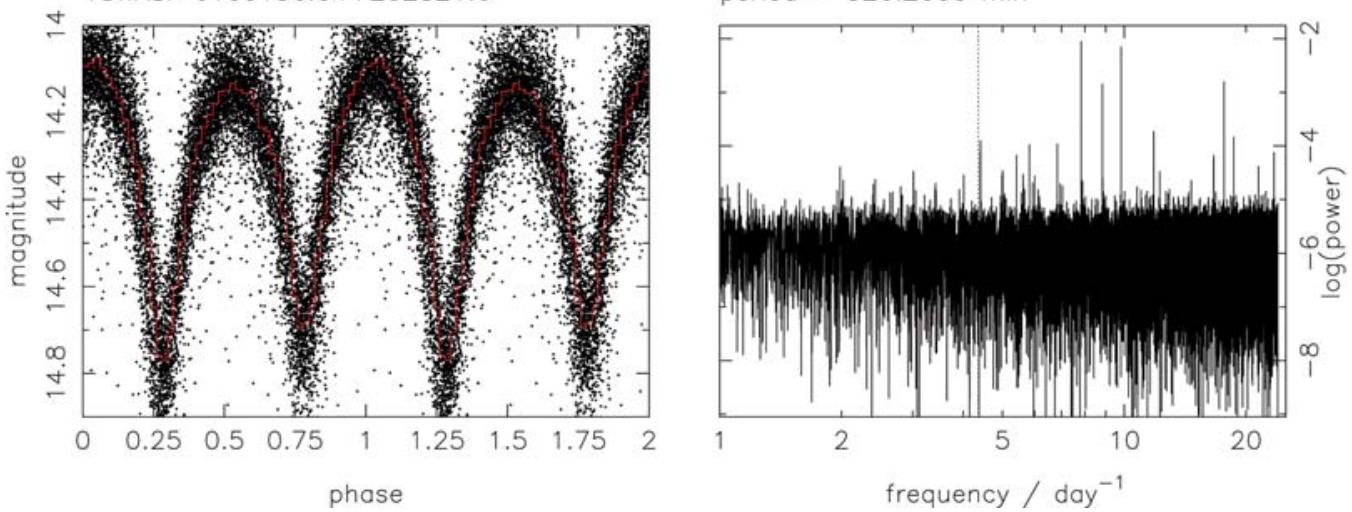

Fig. 2. continued. 

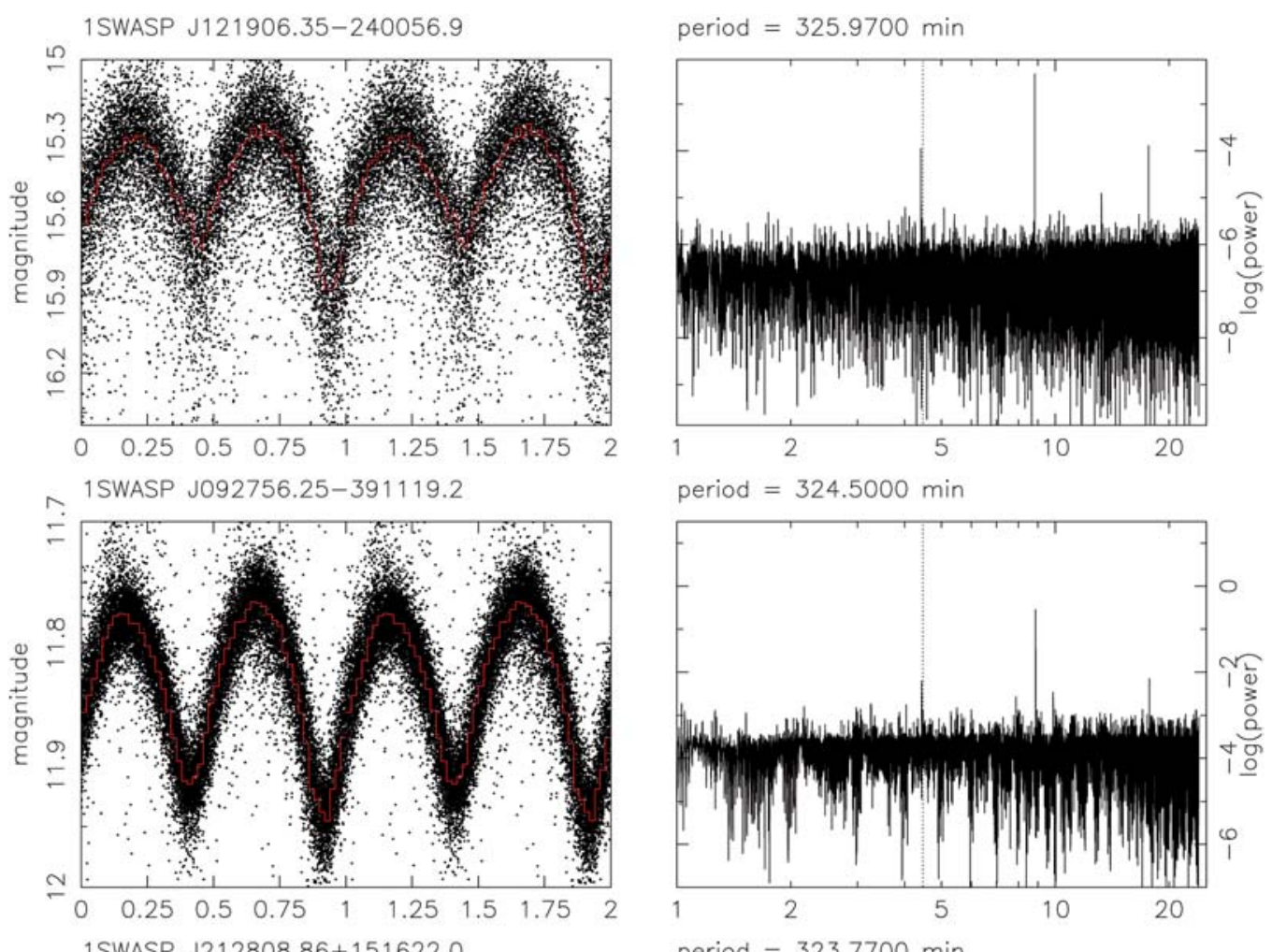

period $=324.5000 \mathrm{~min}$
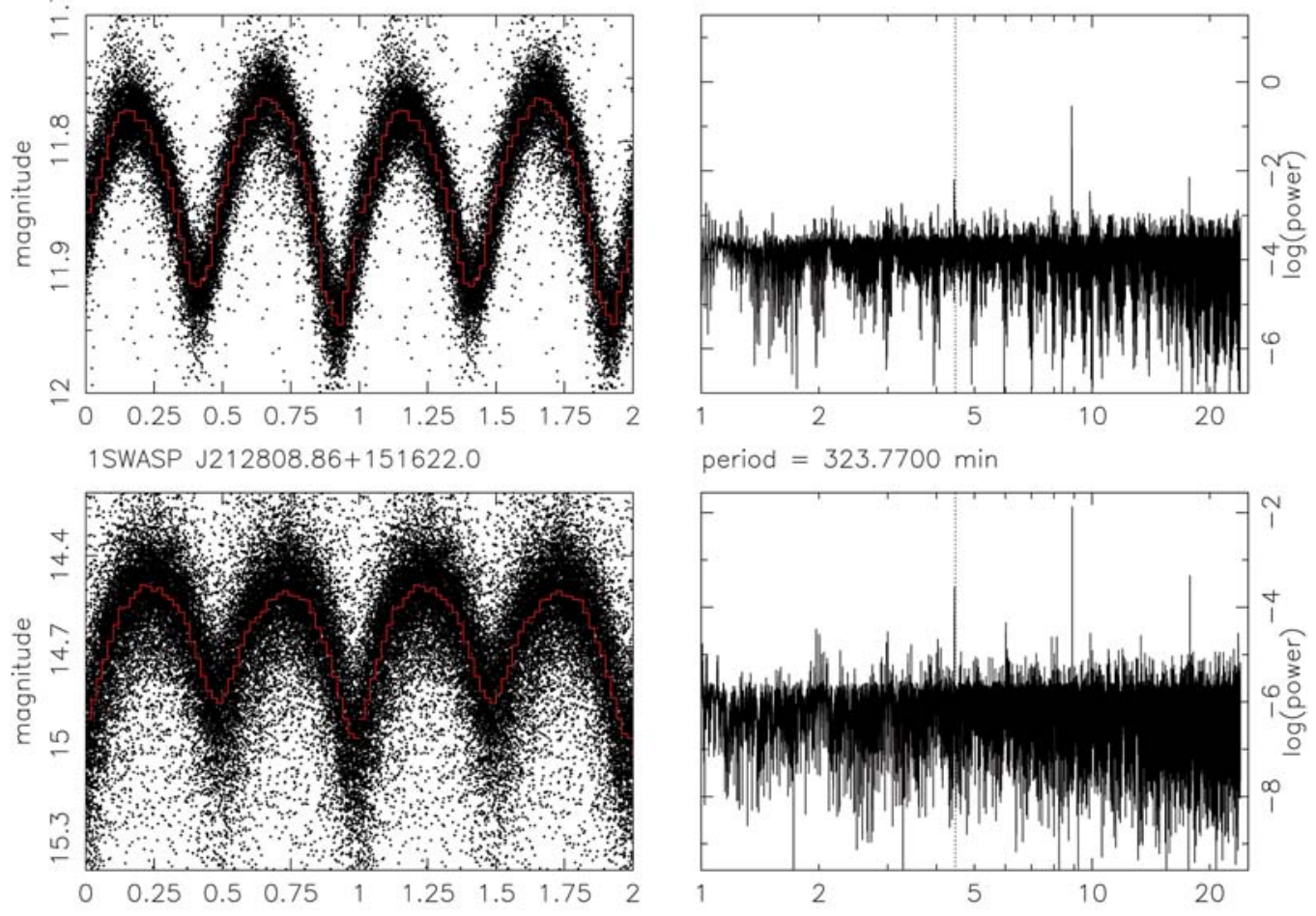

period $=323.7700 \mathrm{~min}$
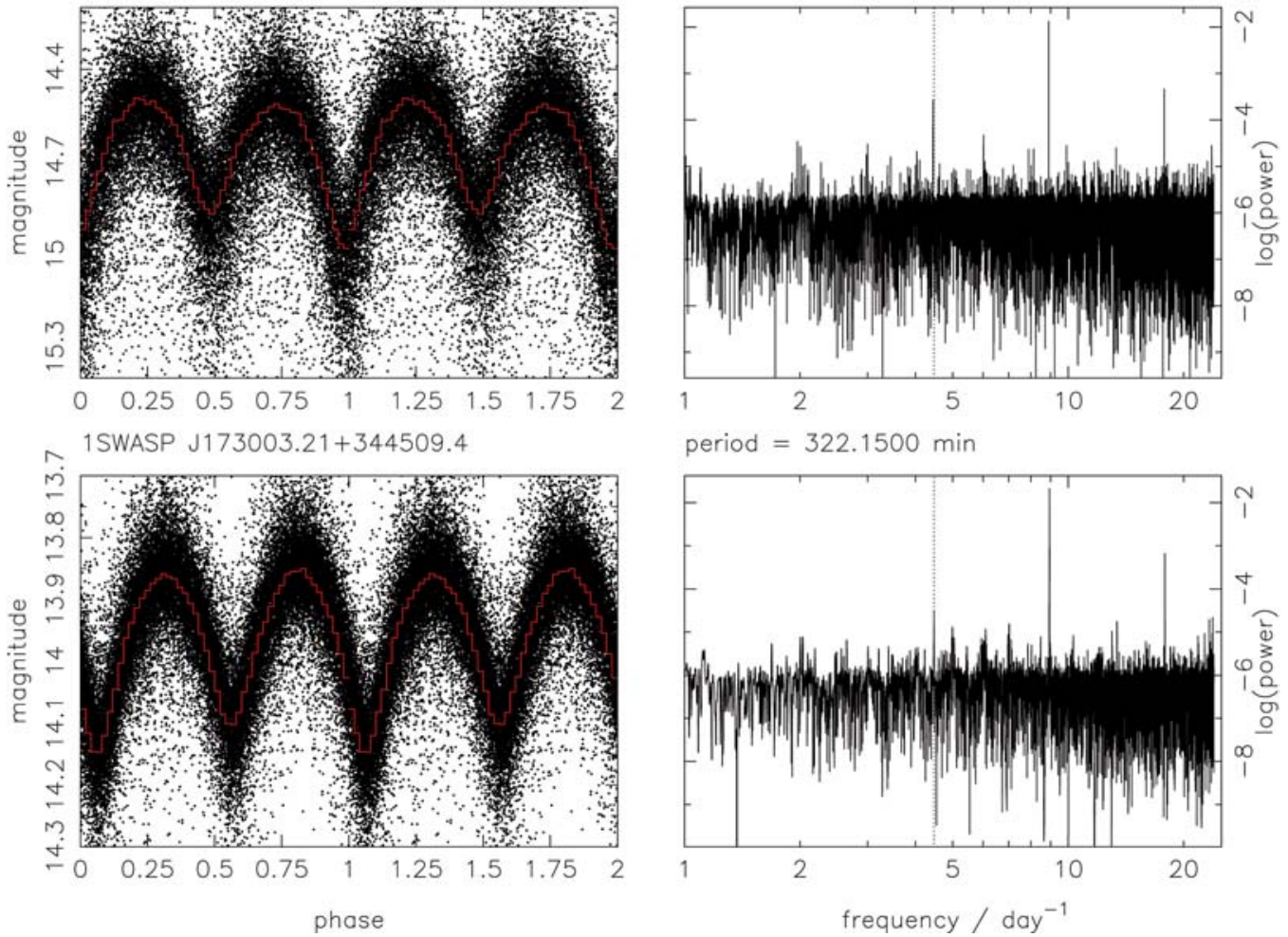

period $=322.1500 \mathrm{~min}$

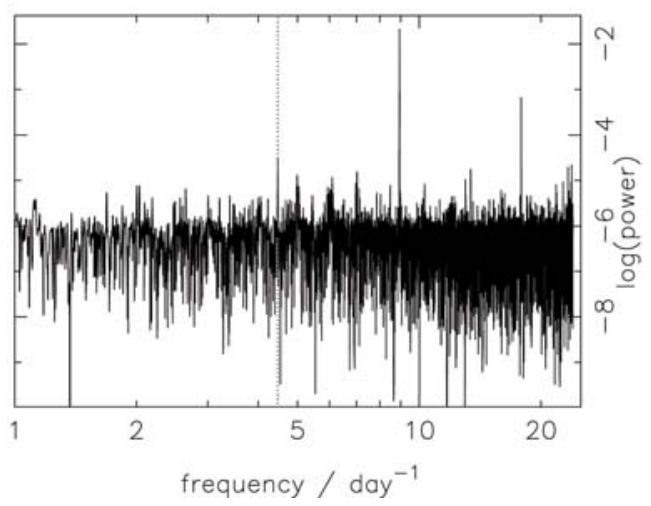

Fig. 2. continued. 
A. J. Norton et al.: WASP short period eclipsing binaries
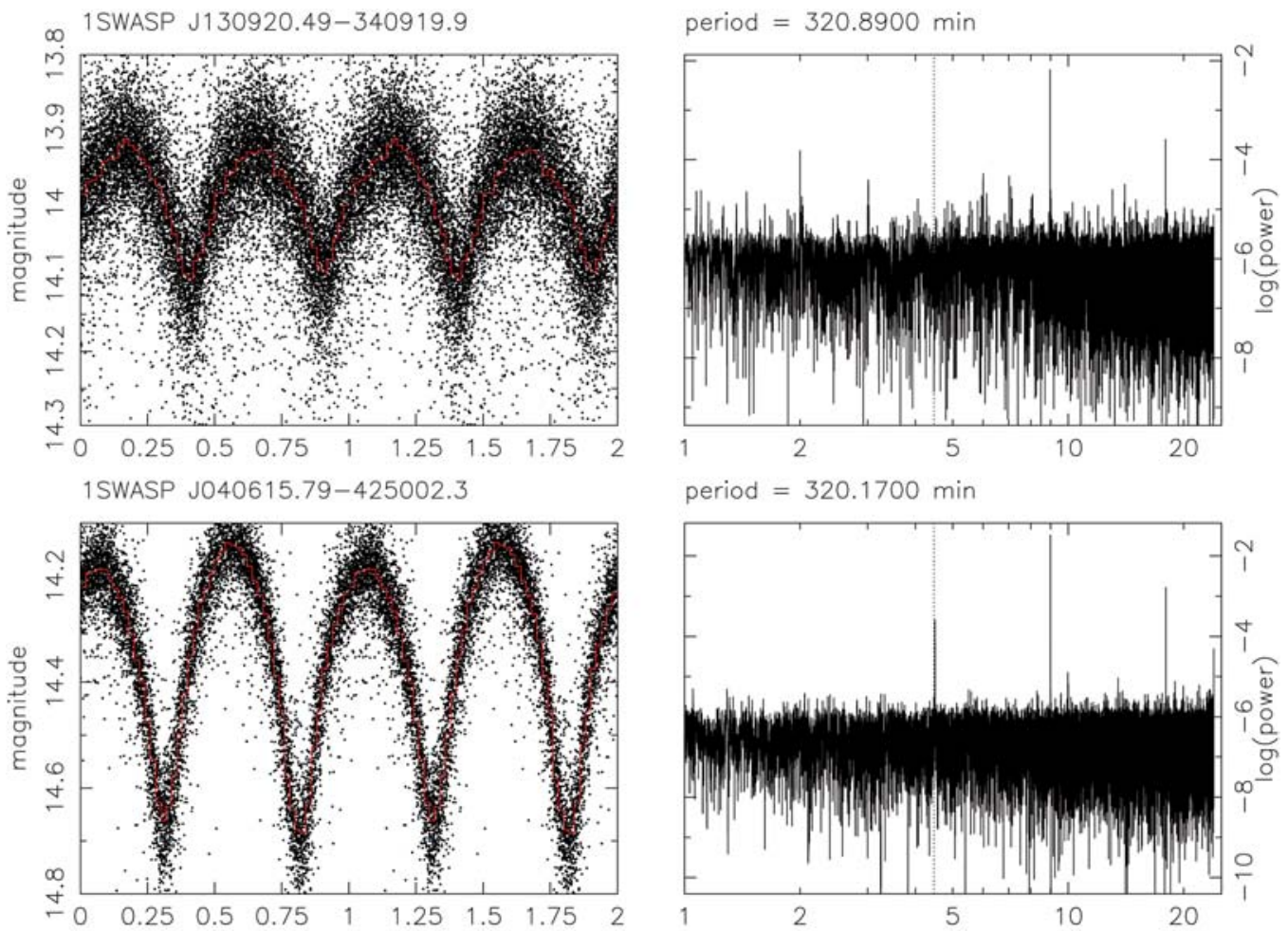

period $=320.1700 \mathrm{~min}$
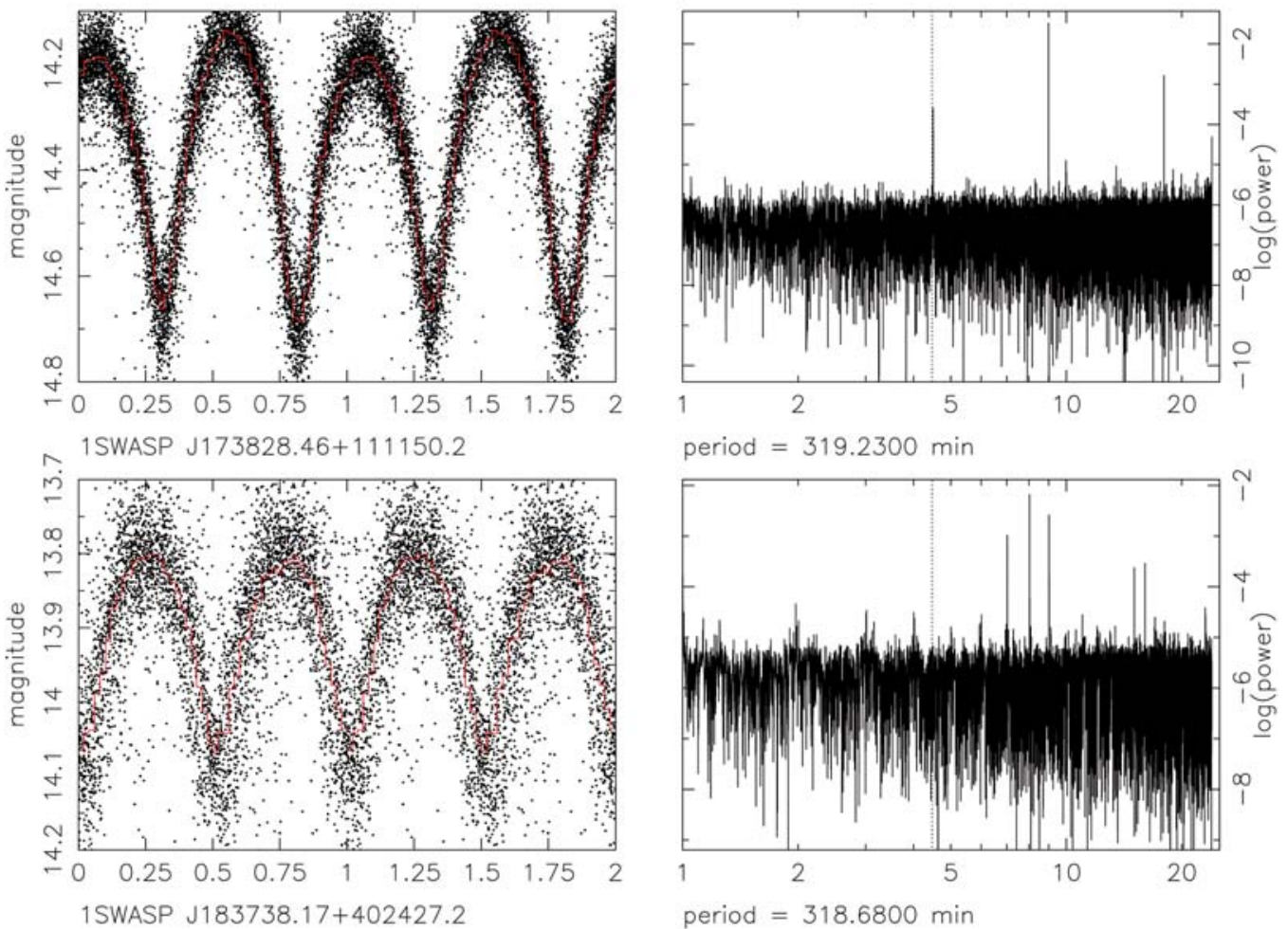

period $=319.2300 \mathrm{~min}$
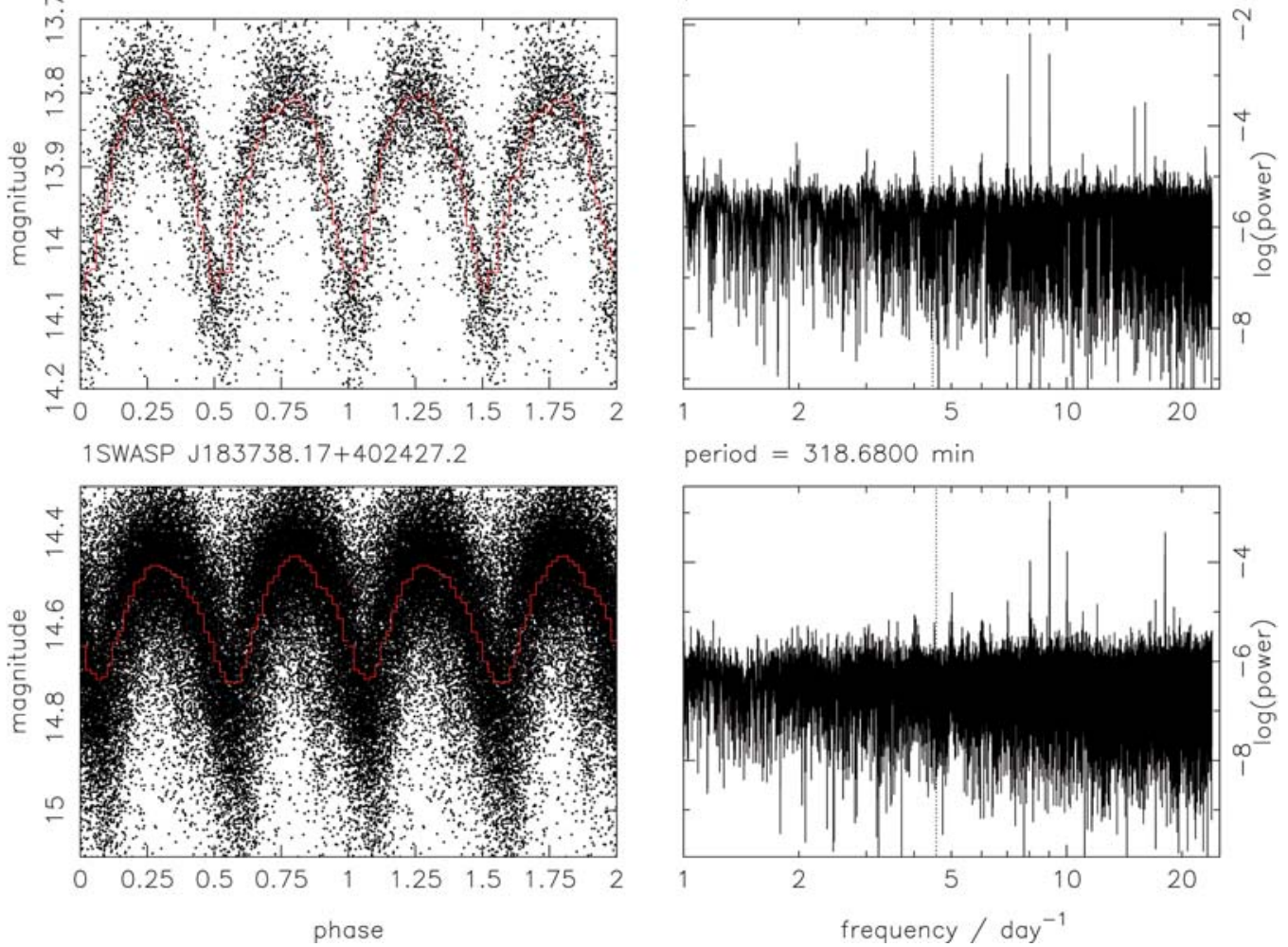

Fig. 2. continued. 

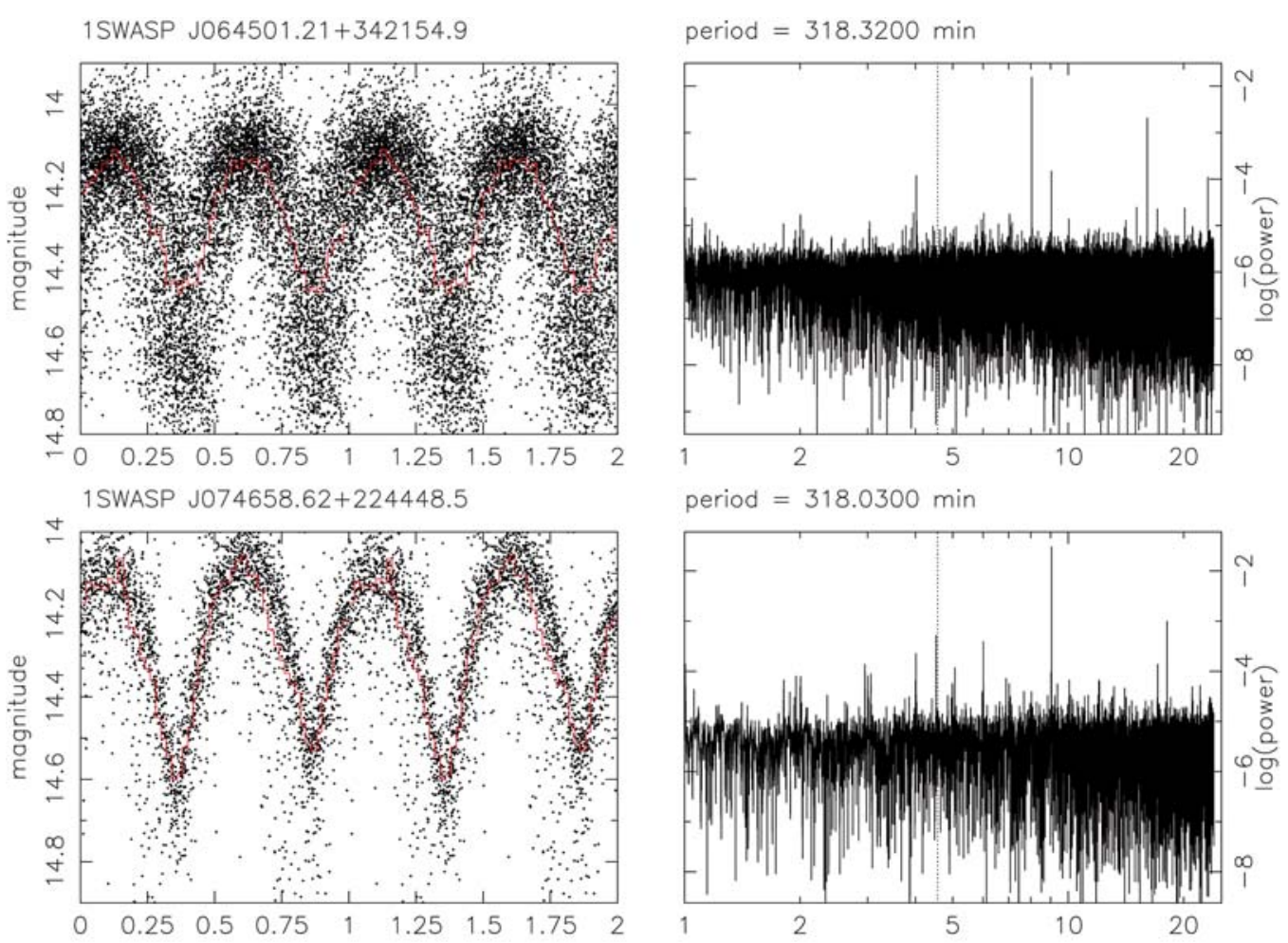

period $=318.0300 \mathrm{~min}$
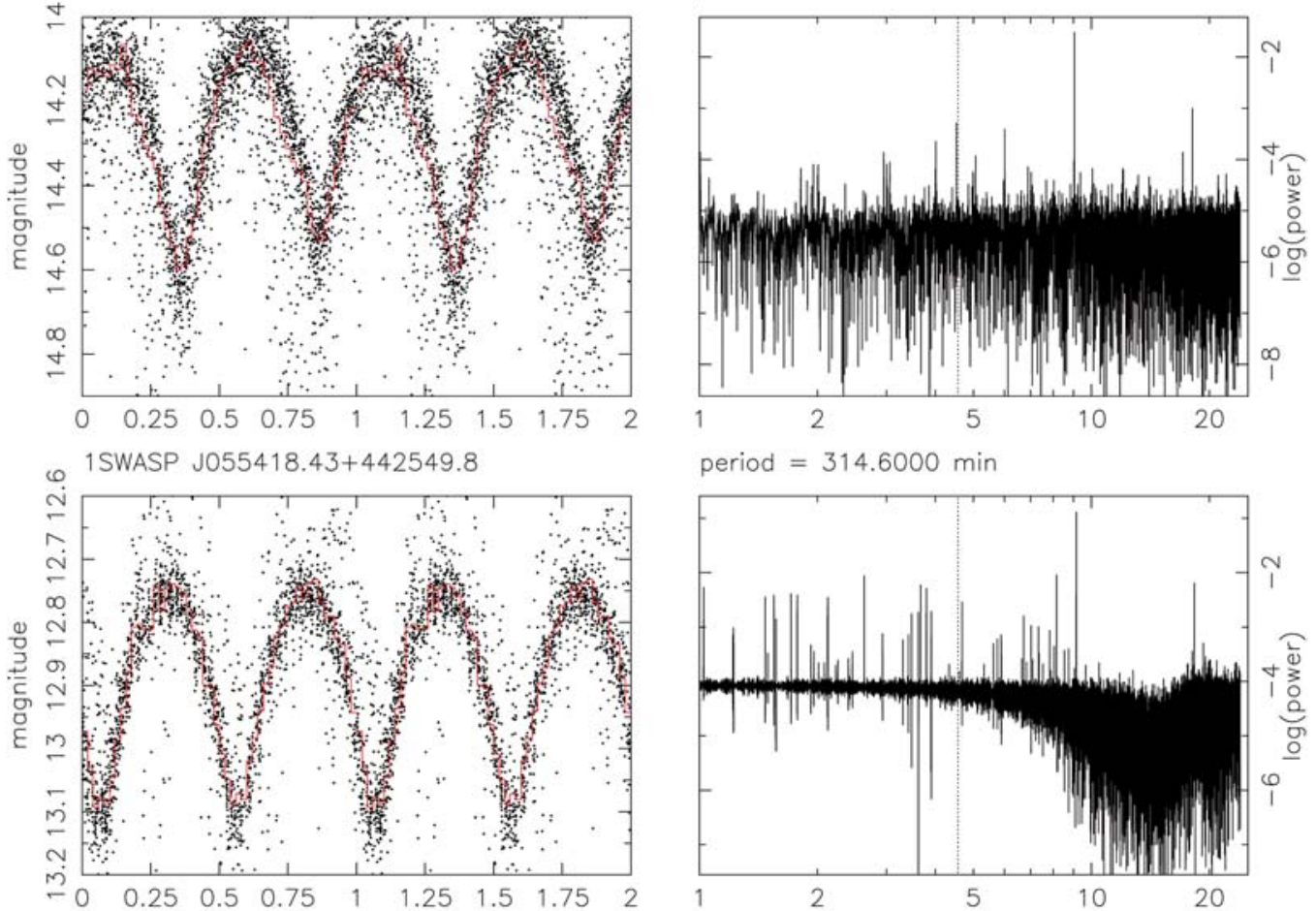

period $=314.6000 \mathrm{~min}$
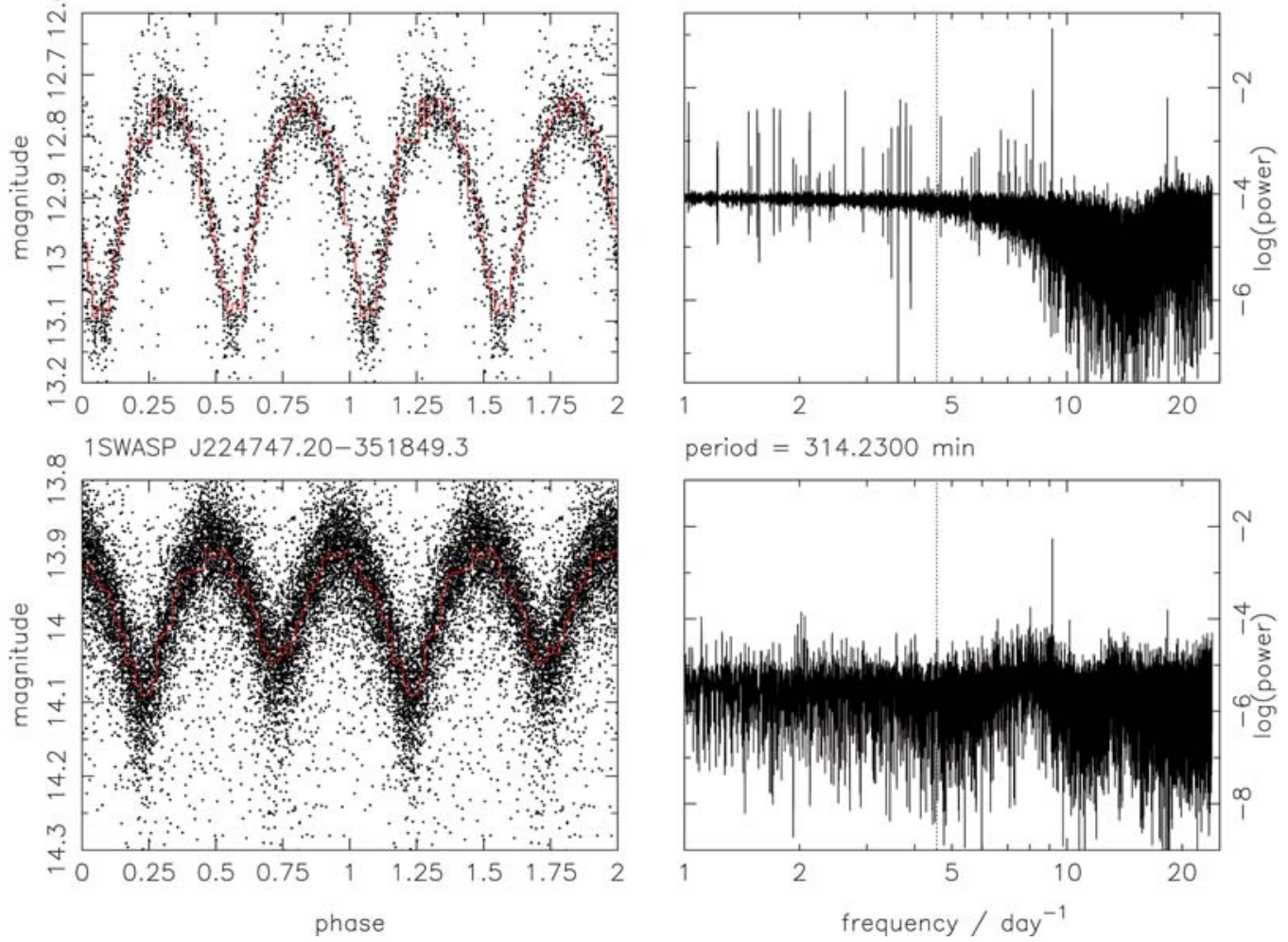

period $=314.2300 \mathrm{~min}$

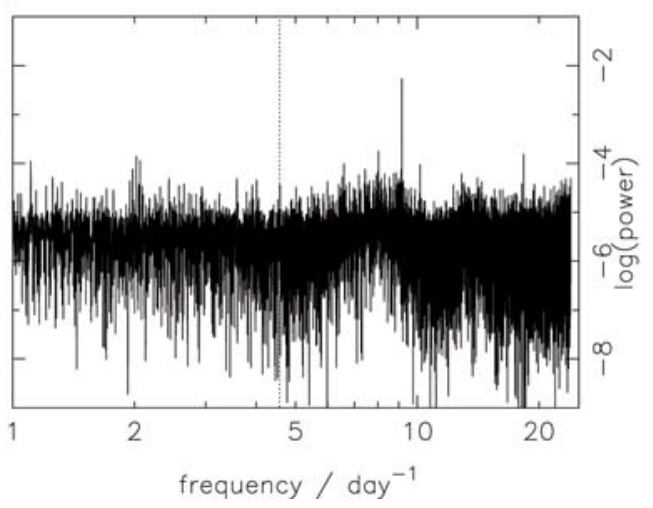

Fig. 2. continued. 
A. J. Norton et al.: WASP short period eclipsing binaries
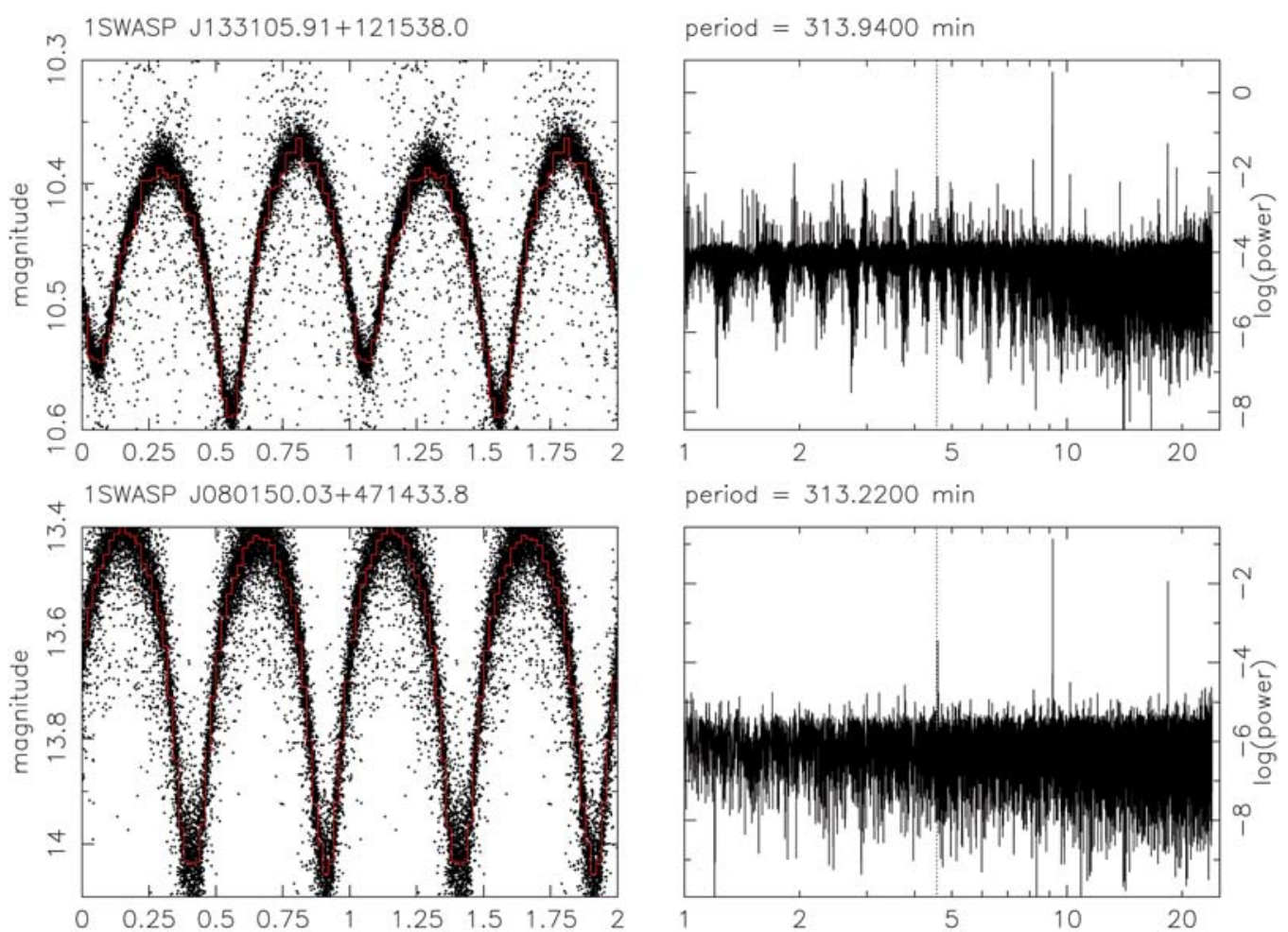

period $=313.2200 \mathrm{~min}$

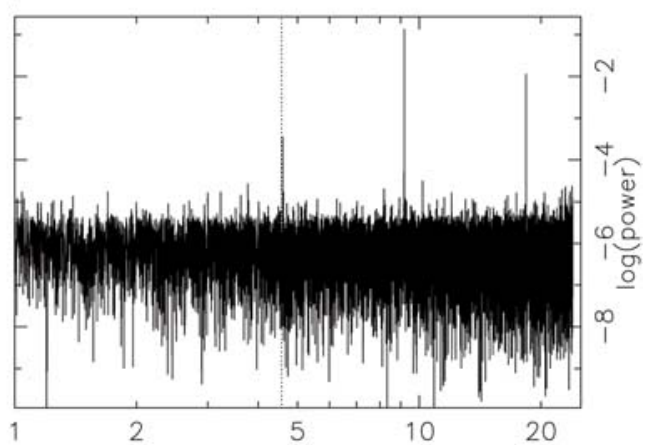

1SWASP J041120.40-230232.3

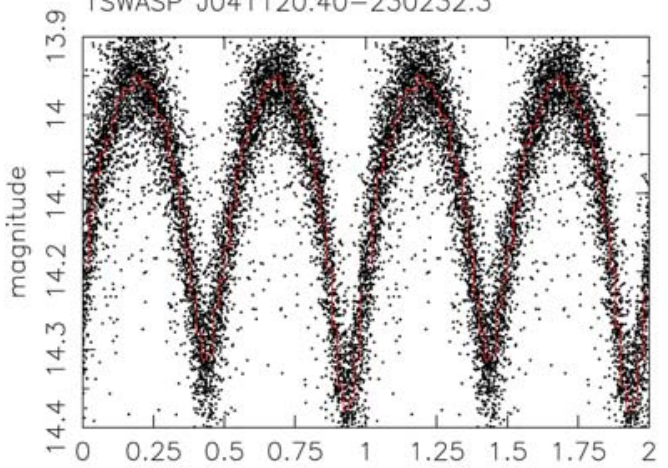

period $=311.5100 \mathrm{~min}$
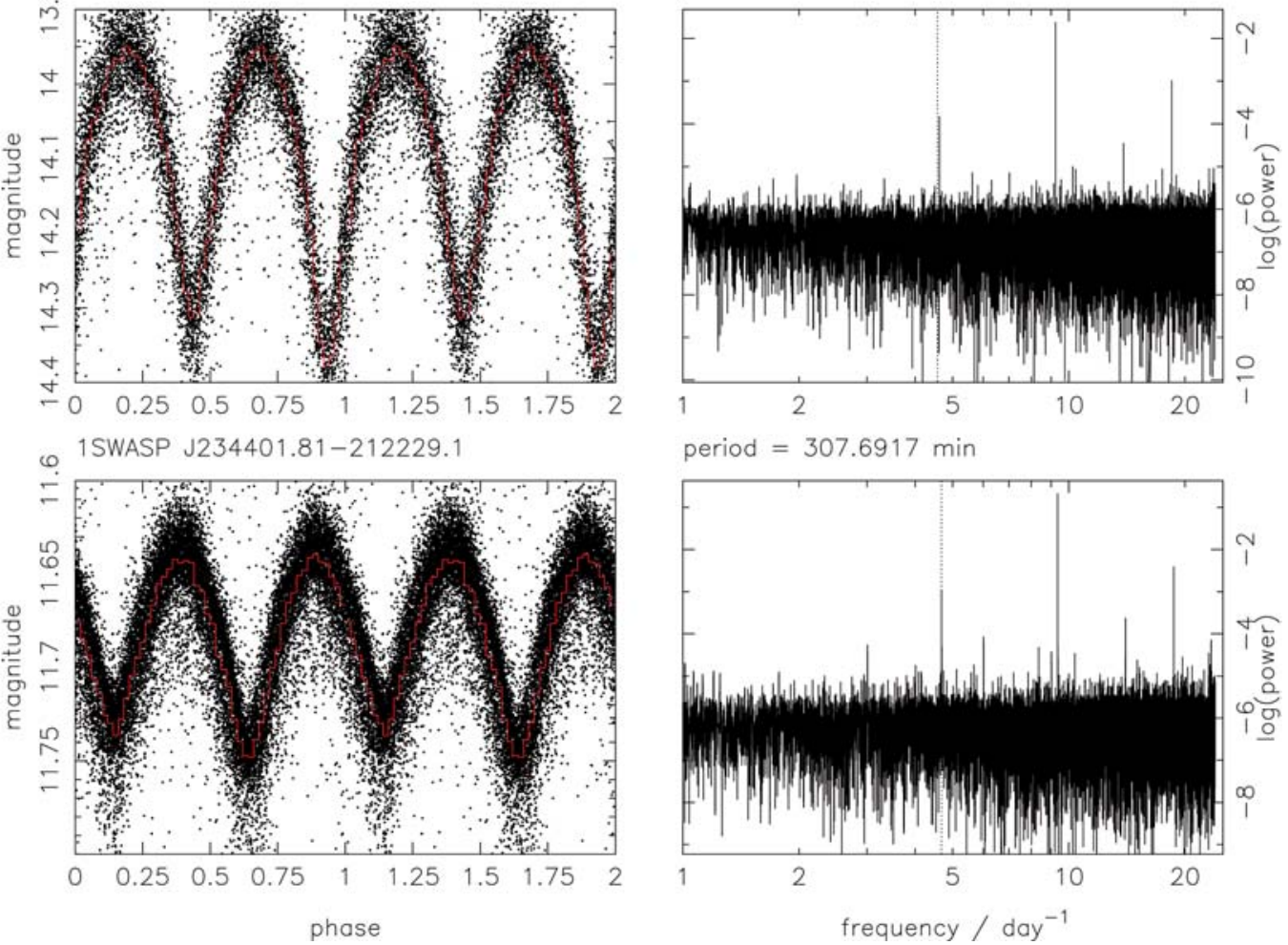

period $=307.6917 \mathrm{~min}$

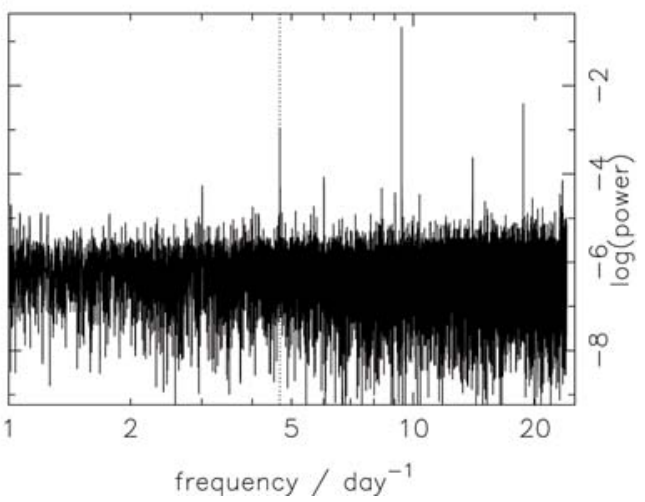

Fig. 2. continued. 

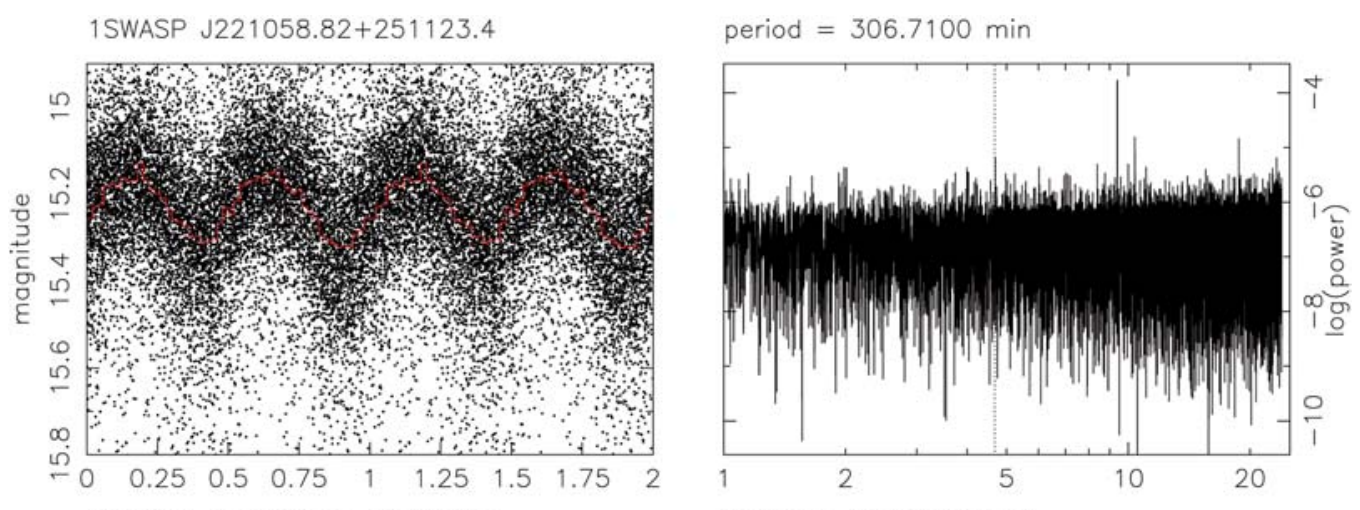

1SWASP J195900.31-252723.1

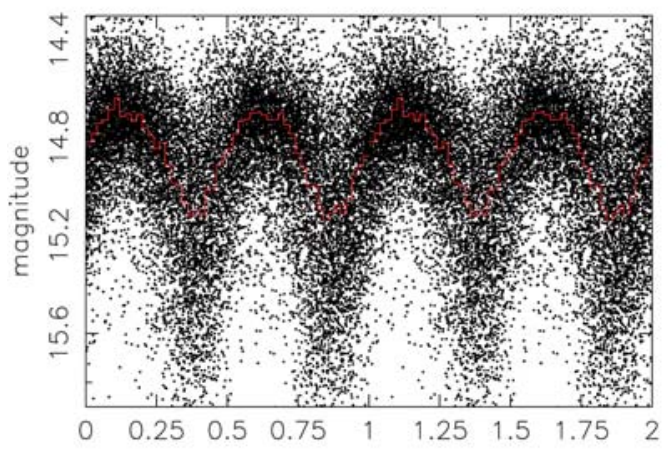

period $=306.3600 \mathrm{~min}$
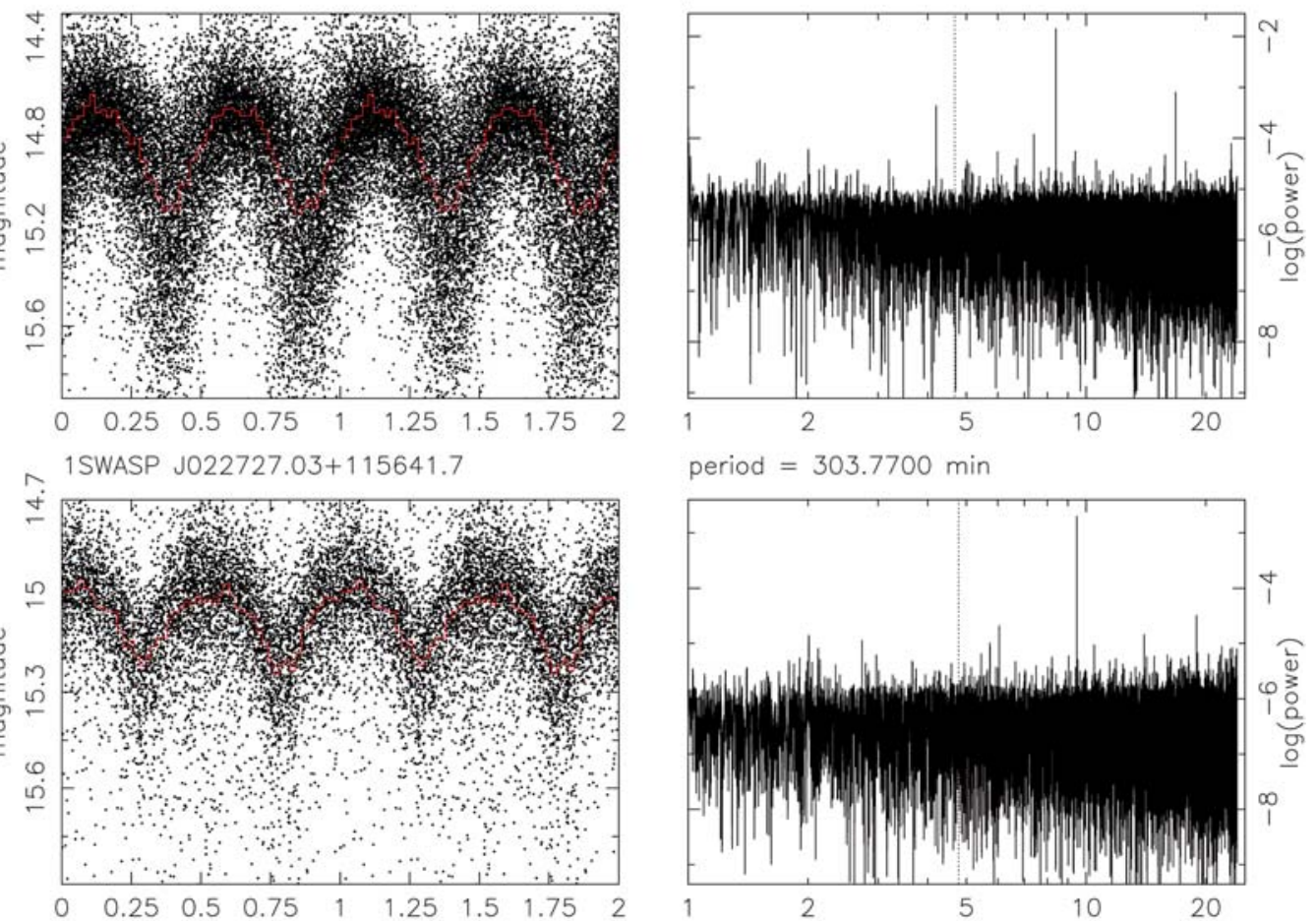

period $=303.7700 \mathrm{~min}$
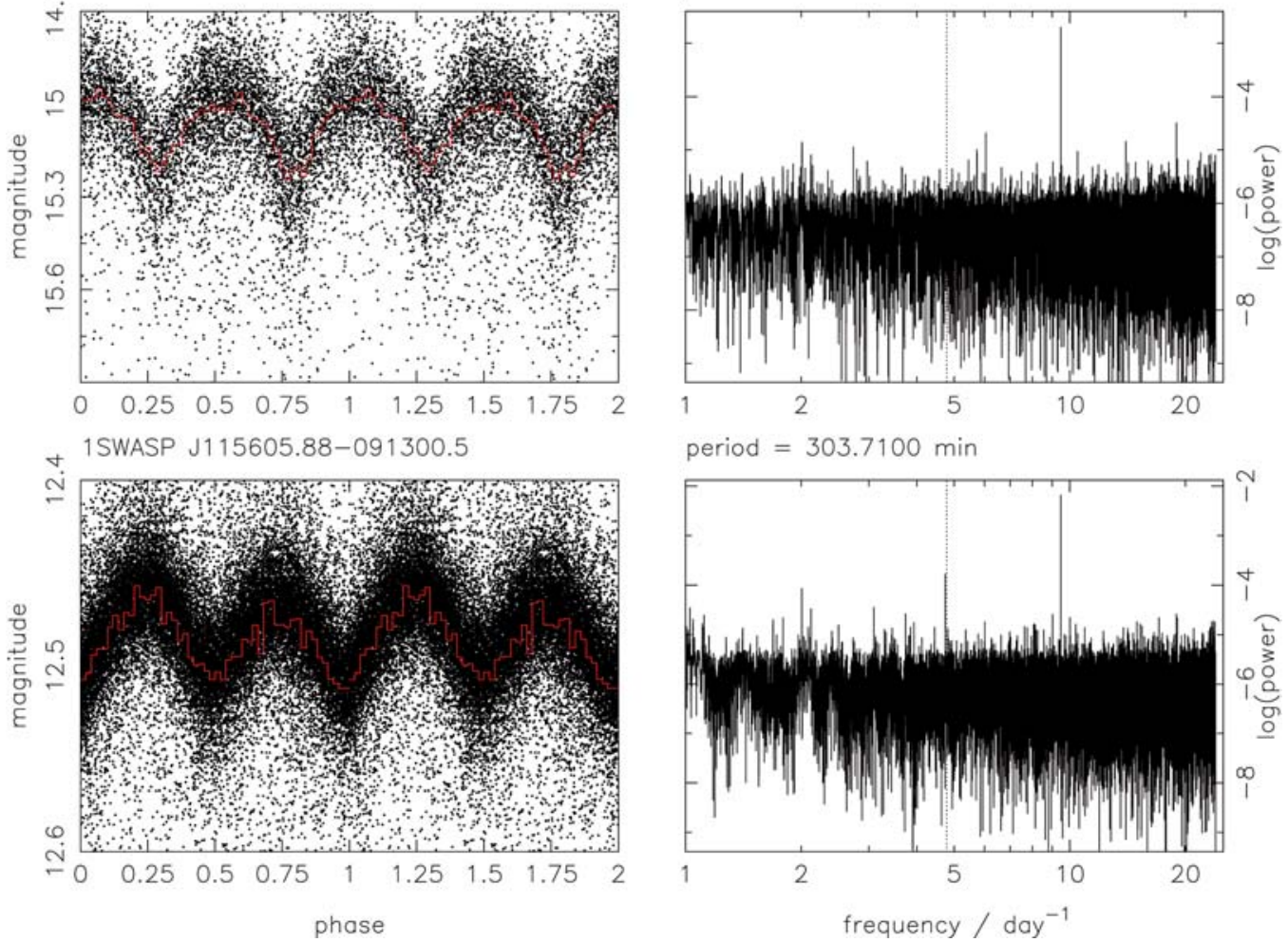

period $=303.7100 \mathrm{~min}$

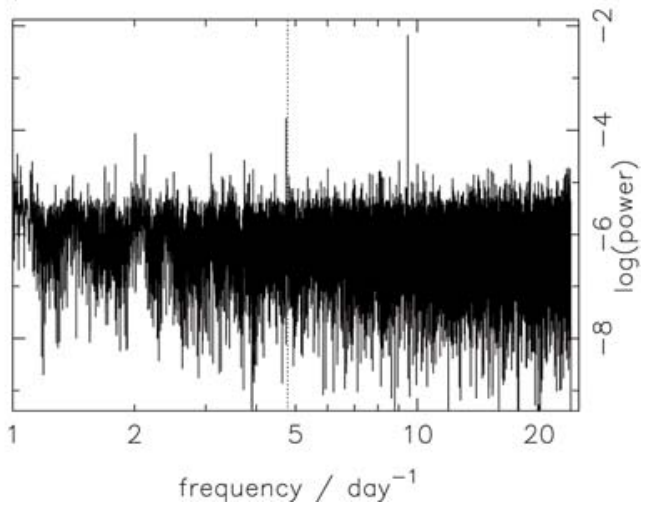

Fig. 2. continued. 
A. J. Norton et al.: WASP short period eclipsing binaries

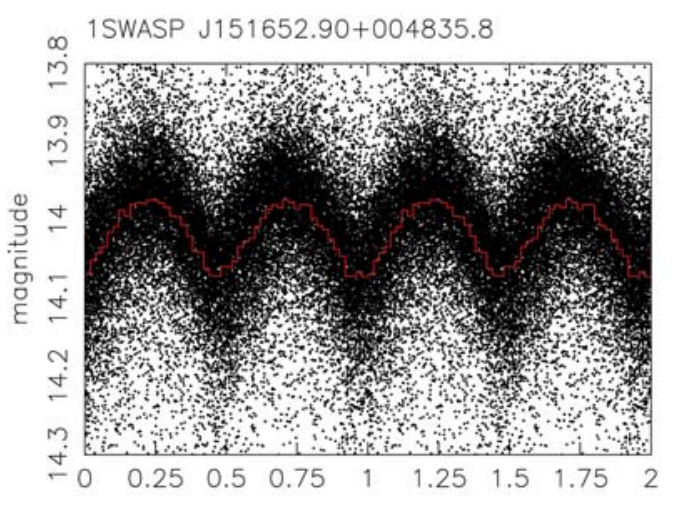

period $=303.4500 \mathrm{~min}$
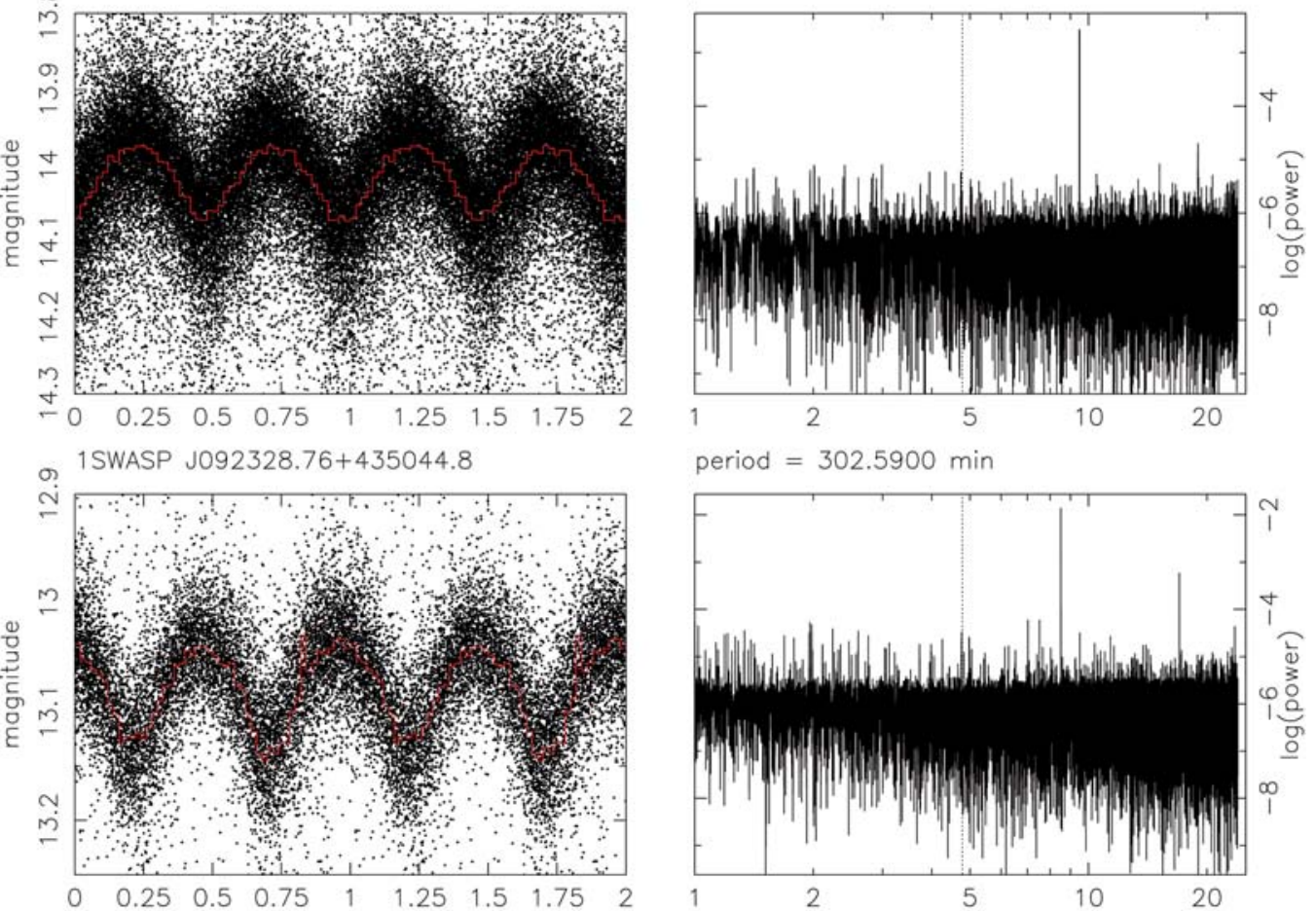

period $=302.5900 \mathrm{~min}$
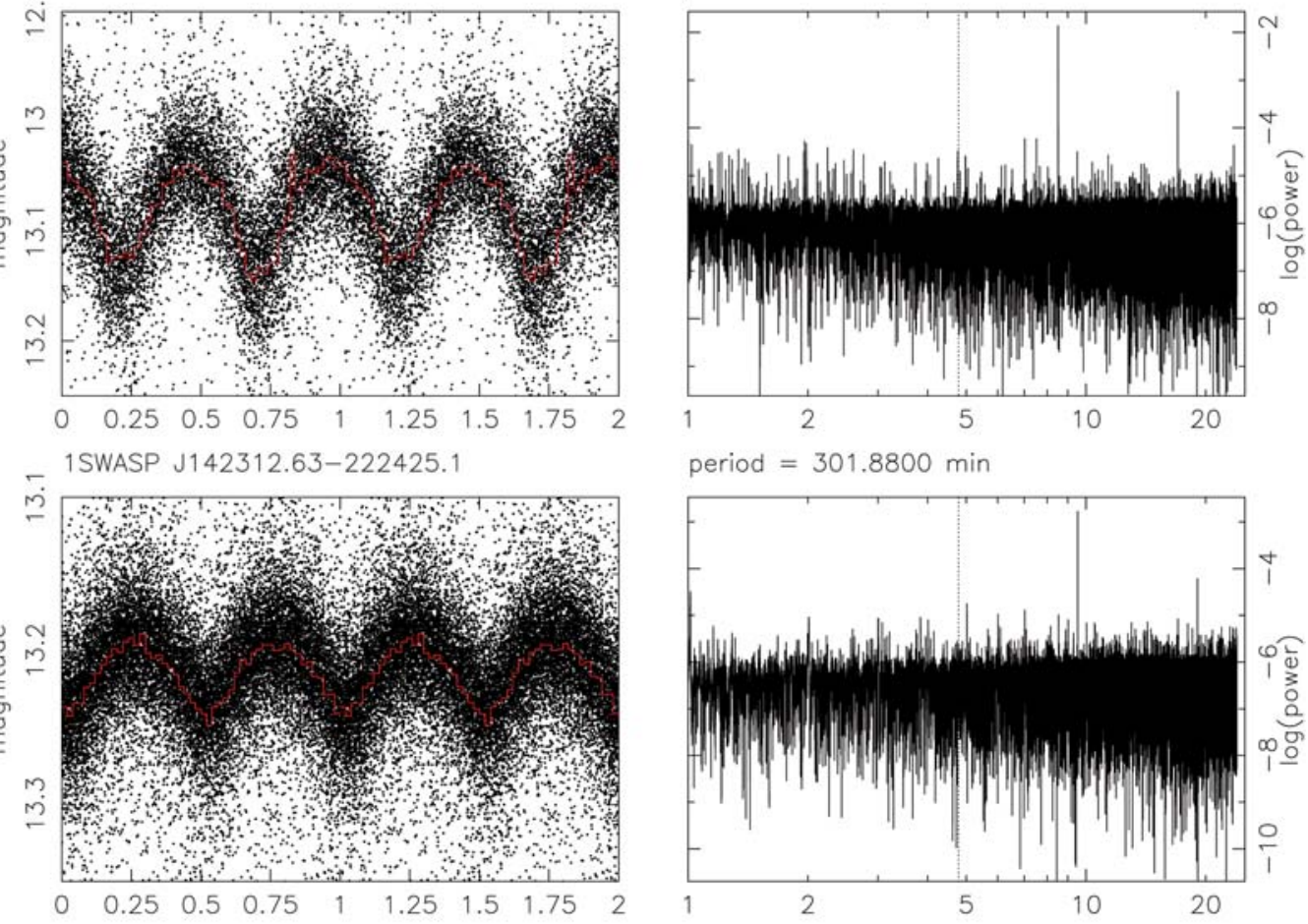

period $=301.8800 \mathrm{~min}$
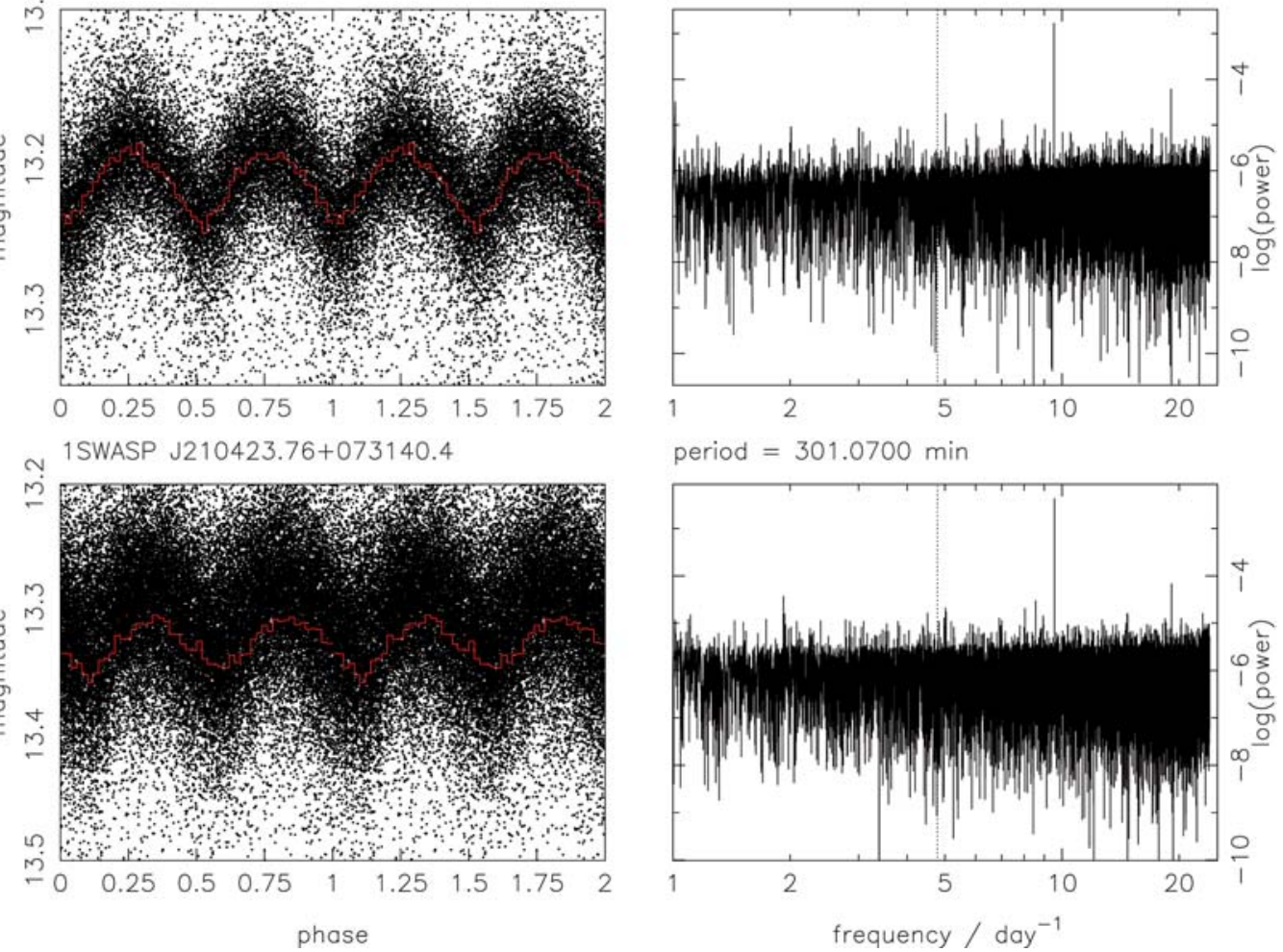

period $=301.0700 \mathrm{~min}$

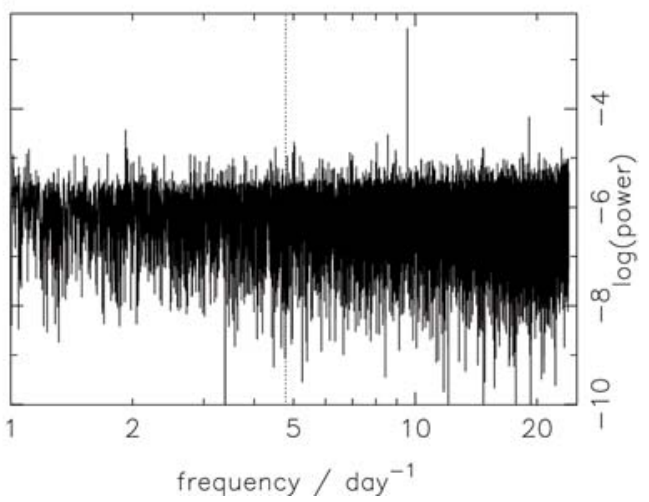

Fig. 2. continued. 